\title{
Kinase-independent functions of RIPK1 regulate hepatocyte survival and liver carcinogenesis
}

\author{
Trieu-My Van, ${ }^{1,2,3}$ Apostolos Polykratis, ${ }^{1,2,3}$ Beate Katharina Straub, ${ }^{4}$ Vangelis Kondylis, ${ }^{1,2,3}$ \\ Nikoletta Papadopoulou, ${ }^{5}$ and Manolis Pasparakis,
}

IInstitute for Cenetics, ${ }^{2}$ Cologne Excellence Cluster on Cellular Stress Responses in Aging-Associated Diseases (CECAD), and ${ }^{3}$ Center for Molecular Medicine (CMMC), University of Cologne, Cologne, Cermany. ${ }^{4}$ Institute of Pathology, University of Mainz, Mainz, Cermany. ${ }^{5}$ Institute for Medical Microbiology, Immunology and Hygiene, Cologne, Cermany.

\begin{abstract}
The mechanisms that regulate cell death and inflammation play an important role in liver disease and cancer. Receptorinteracting protein kinase 1 (RIPK1) induces apoptosis and necroptosis via kinase-dependent mechanisms and exhibits kinase-independent prosurvival and proinflammatory functions. Here, we have used genetic mouse models to study the role of RIPK1 in liver homeostasis, injury, and cancer. While ablating either RIPK1 or RelA in liver parenchymal cells (LPCs) did not cause spontaneous liver pathology, mice with combined deficiency of RIPK1 and RelA in LPCs showed increased hepatocyte apoptosis and developed spontaneous chronic liver disease and cancer that were independent of TNF receptor 1 (TNFR1) signaling. In contrast, mice with LPC-specific knockout of Ripk1 showed reduced diethylnitrosamine-induced (DEN-induced) liver tumorigenesis that correlated with increased DEN-induced hepatocyte apoptosis. Lack of RIPK1 kinase activity did not inhibit DEN-induced liver tumor formation, showing that kinase-independent functions of RIPK1 promote DEN-induced hepatocarcinogenesis. Moreover, mice lacking both RIPK1 and TNFR1 in LPCs displayed normal tumor formation in response to DEN, demonstrating that RIPK1 deficiency decreases DEN-induced liver tumor formation in a TNFR1-dependent manner. Therefore, these findings indicate that RIPK1 cooperates with NF- KB signaling to prevent TNFR1-independent hepatocyte apoptosis and the development of chronic liver disease and cancer, but acts downstream of TNFR1 signaling to promote DENinduced liver tumorigenesis.
\end{abstract}

\section{Introduction}

Liver injury in response to immune deregulation and/or infection can result in acute liver failure or chronic liver disease. Chronic hepatocyte death coupled with compensatory hepatocyte proliferation greatly increases the risk of tumorigenesis. Indeed, hepatocellular carcinoma (HCC), the most frequent primary liver cancer, usually develops in the context of chronic liver injury and inflammation caused by hepatitis B virus (HBV) or HCV infections, alcoholic and nonalcoholic steatohepatitis, and aflatoxin-mediated toxicity (1). HCC is one of the most frequent and difficult to treat types of cancer, constituting the third most common cause of cancer-related death worldwide $(1,2)$.

Death receptors activate distinct signaling pathways controlling inflammation and cell death and are implicated in liver disease and cancer $(3,4)$. Receptor-interacting protein kinase 1 (RIPK1) is a key regulator of TNF receptor 1 (TNFR1) signaling (5). The kinase activity of RIPK1 triggers cell death by activating either caspase-8-mediated apoptosis or RIPK3-MLKL-dependent (where MLKL indicates mixed lineage kinase domain-like protein) necroptosis. In addition, RIPK1 induces prosurvival and proinflammatory responses via kinase-independent scaffolding functions (5). RIPK1 is recruited to the TNFR1 signaling complex together with TNFR1-associated death domain protein (TRADD),

Conflict of interest: The authors have declared that no conflict of interest exists Submitted: December 28, 2016; Accepted: March 29, 2017.

Reference information: / Clin Invest. 2017;127(7):2662-2677. https://doi.org/10.1172/JCI92508. the E3 ubiquitin ligases TNF receptor-associated factor 2 (TRAF2) and cellular inhibitors of apoptosis 1 (cIAP1) and cIAP2, and the linear ubiquitin chain assembly complex (LUBAC) $(6,7)$. RIPK1 contributes to the activation of $\mathrm{NF}-\kappa \mathrm{B}$, but this function seems to be dependent on the cell types and experimental conditions studied (8-11). NF- $\mathrm{KB}$ signaling regulates immune and inflammatory responses and cell survival $(12,13)$ and is implicated in the pathogenesis of hepatitis and HCC $(14,15)$. Mice with liver parenchymal cell-specific (LPC-specific) ablation of NF- $\mathrm{KB}$ essential modulator (NEMO) /I $\mathrm{B}$ kinase $\gamma(\mathrm{IKK} \gamma)$, the regulatory subunit of the IKK complex that is required for canonical NF- $\kappa B$ activation, developed spontaneously chronic hepatitis and HCC due to RIPK1 kinase activity-driven, FAS-associated death domain (FADD)/ caspase-8-mediated hepatocyte apoptosis (16-19). In contrast, LPC-specific NF-кB deficiency did not cause spontaneous development of severe chronic liver disease and cancer, suggesting that NEMO regulates liver homeostasis by NF- $\mathrm{KB}$-dependent and -independent functions (16).

Mice lacking RIPK1 specifically in LPCs (Ripk1 $1^{f / f l}$ Alfp-Cre mice, hereafter referred to as RIPK $1^{\text {LPC-KO }}$ mice) did not develop spontaneous liver disease, showing that RIPK1 is not required for normal liver homeostasis under steady-state conditions $(16,20-$ 24). However, RIPK1 $1^{\mathrm{LPC}-\mathrm{KO}}$ mice were highly sensitive to concanavalin A-mediated (ConA-mediated) liver damage, which was partly induced by TNF-mediated hepatocyte apoptosis (24). Moreover, administration of LPS caused severe liver injury in RIPK1 $1^{\text {LPC-KO }}$ mice by inducing TNF-dependent hepatocyte apoptosis $(20,21)$. Interestingly, although LPC-specific deficiency of either RIPK1 
or TRAF2 (Traf $2^{f / f l}$ Alfp-Cre mice) did not cause spontaneous liver injury, mice with combined ablation of RIPK1 and TRAF2 in LPCs developed severe chronic cholestatic liver disease that resulted in the development of liver tumors by the age of 1 year (20). LPCspecific knockout of caspase- 8 fully prevented liver damage and tumorigenesis in these mice, showing that combined loss of RIPK1 and TRAF2 sensitizes hepatocytes to caspase-8-dependent apoptosis that drives chronic liver disease and tumorigenesis (20).

RIPK1 has also been implicated in the regulation of cellular responses to genotoxic stress. RIPK1 was suggested to be important for NEMO-dependent activation of prosurvival NF- $\kappa \mathrm{B}$ signaling in response to DNA damage (25). Moreover, genotoxic stress was proposed to trigger the formation of the ripoptosome, inducing RIPK1 kinase activity-dependent cell death either directly or indirectly via autocrine TNF production $(26,27)$. The involvement of RIPK1 in determining the cell fate in response to genotoxic stress and proinflammatory signaling suggests that RIPK1 may be critically involved in carcinogenesis.

Here, we studied the biological significance of RIPK1 kinasedependent and -independent functions in the liver using genetic mouse models. Our results revealed important functions of RIPK1 in the regulation of hepatocyte survival, liver homeostasis, and hepatocarcinogenesis.

\section{Results}

RIPK1 prevents LPS-induced liver injury by inhibiting TNF-induced hepatocyte apoptosis. To study the role of RIPK1 in the liver, we generated mice with LPC-specific RIPK1 knockout (RIPK1 ${ }^{\text {LPC-KO})}$ by crossing mice carrying loxP-flanked Ripk1 alleles (8) with AlfpCre transgenics, which mediate efficient Cre recombination in hepatocytes and biliary epithelial cells, but not in endothelial or Kupffer cells (28). RIPK $1^{\text {LPC-KO }}$ mice were born at the expected Mendelian frequency and did not show signs of liver pathology (16). To address the role of RIPK1 in liver responses to endotoxic shock, we injected RIPK1 $1^{\text {LPC-KO }}$ and Ripk $1^{f / f l}$ littermates with a low dose of LPS ( $5 \mu \mathrm{g} / \mathrm{g}$ of body weight [BW]). All RIPK1 $1^{\mathrm{LPC}-\mathrm{KO}}$ mice became terminally ill and were sacrificed within 6 hours after LPS injection, in contrast with their Ripk $1^{f l f l}$ littermates, which all survived (Figure 1A). RIPK1 ${ }^{\text {LPC-KO }}$ mice showed strongly elevated levels of serum alanine aminotransferase (ALT) 5.5 hours after LPS injection, indicative of severe liver damage (Figure 1B). Consistently, immunostaining of liver sections with antibodies against cleaved caspase-3 (CC3) as well as immunoblot analysis of liver protein extracts for CC 3 and cleaved PARP1 revealed increased hepatocyte apoptosis in livers from LPS-injected RIPK1 $1^{\text {LPC-KO }}$ mice (Figure 1, C and D). LPS administration induced similar levels of TNF expression in the serum of RIPK $1^{\text {LPC-KO }}$ and Ripk $1^{f / f l}$ mice, suggesting that the increased liver damage in RIPK1 $1^{\mathrm{LPC}-\mathrm{KO}}$ mice was not due to higher TNF levels, but rather to the sensitivity of RIPK1-deficient hepatocytes to TNF-induced apoptosis (Figure 1E). To assess the mechanism of LPS-induced apoptosis, we prepared primary hepatocytes from RIPK1 $1^{\mathrm{LPC}-\mathrm{KO}}$ and Ripk $1^{f l / f l}$ mice and observed that RIPK1-deficient hepatocytes died spontaneously within 24 hours after isolation (Figure $1 F)$. Treatment with the pan-caspase inhibitor Z-VAD-FMK prevented the spontaneous death of RIPK1-deficient hepatocytes, suggesting they die by apoptosis. Furthermore, neutralizing anti- bodies against TNF partially inhibited spontaneous apoptosis of RIPK1-deficient primary hepatocytes, while anti-FasL antibodies had no effect, even when given together with anti-TNF (Figure $1 \mathrm{~F}$ ). These results suggest that TNF produced by liver cells during preparation and early in vitro culture drives apoptosis of RIPK1-deficient primary hepatocytes.

Because NF- $\kappa \mathrm{B}$ inhibition sensitizes hepatocytes to LPS/ TNF-induced apoptosis (16), we assessed TNF-induced NF- $\kappa B$ activation in primary hepatocytes from RIPK1 $1^{\mathrm{LPC}-\mathrm{KO}}$ and Ripk $1^{f / f l}$ mice. To prevent the spontaneous death of RIPK1-deficient hepatocytes, we cultured them in the presence of Z-VAD-FMK. TNF stimulation caused rapid phosphorylation and degradation of I $\mathrm{B}$ and robust nuclear accumulation of RelA in Ripk $1^{f l / f l}$ hepatocytes, while RIPK1-deficient hepatocytes showed impaired NF-кB activation (Figure 1, $\mathrm{G}$ and $\mathrm{H}$ ). In addition, RIPK1 deficiency reduced the TNF-induced expression of the NF- $\mathrm{BB}$-dependent genes TNF alpha induced protein 3 (Tnfaip3), baculoviral IAP repeat-containing 3 (Birc3), nuclear factor of kappa light polypeptide gene enhancer in B cells inhibitor, alpha (Nfkbia), and Tnf (Figure 1I). Therefore, RIPK1 deficiency partially inhibited TNF-mediated $\mathrm{NF}-\kappa \mathrm{B}$ activation in hepatocytes, suggesting that impaired NF- $\mathrm{B}$ signaling contributes to the increased sensitivity of RIPK1-deficient hepatocytes to apoptosis.

Our in vitro experiments in primary hepatocytes suggested that LPS could cause liver damage in RIPK1 ${ }^{\mathrm{LPC}-\mathrm{KO}}$ mice by triggering TNFR1-induced hepatocyte apoptosis. To address the role of TNFR1-mediated hepatocyte apoptosis in RIPK1 ${ }^{\text {LPC-KO }}$ mice, we crossed them with Tnfri $1^{f / f l}$ (29), Tradd $d^{l / f l}$ (30), and Fadd ${ }^{l / f l}$ (31) mice to generate RIPK1 $1^{\mathrm{LPC}-\mathrm{KO}}$ mice that additionally lacked TNFR1, TRADD, or FADD in LPCs (Supplemental Figure 1A; supplemental material available online with this article; https://doi.org/10.1172/ JCI92508DS1). LPC-specific ablation of FADD fully protected RIPK1 $1^{\text {LPC-KO }}$ mice from LPS-induced hepatocyte death and liver damage, showing that LPS triggers liver failure in these mice by inducing FADD-caspase-8-dependent hepatocyte apoptosis (Figure 1, J and K, and Supplemental Figure 1, B and C). LPS-induced hepatocyte apoptosis in RIPK1 $1^{\mathrm{LPC}-\mathrm{KO}}$ mice was also fully prevented by LPC-specific ablation of TNFR1, demonstrating that LPS causes liver damage indirectly by triggering the expression of TNF that acts via TNFR1 to kill RIPK1-deficient hepatocytes (Figure 1, J and K, and Supplemental Figure 1, B and C). Moreover, LPCspecific Tradd knockout also protected RIPK1 ${ }^{\mathrm{LPC}-\mathrm{KO}}$ mice from LPSinduced liver damage, showing that the TNF-induced activation of caspase-8-mediated apoptosis in RIPK1-deficient hepatocytes was dependent on TRADD (Figure 1, J and K, and Supplemental Figure 1, B and C). Therefore, LPS administration in vivo caused liver failure in RIPK1 $1^{\mathrm{LPC}-\mathrm{KO}}$ mice by triggering TNFR1-induced, TRADD-mediated activation of FADD-caspase-8-dependent apoptosis of RIPK1-deficient hepatocytes.

We then assessed the role of TNFR1, TRADD, and FADD in spontaneous apoptosis of RIPK1-deficient primary hepatocytes. Primary hepatocytes from RIPK1 $1^{\mathrm{LPC}-\mathrm{KO}} \mathrm{FADD}^{\mathrm{LPC}-\mathrm{KO}}$ mice had viability similar to that of control Ripk $1^{f l f l}$ hepatocytes, showing that RIPK1-deficient hepatocytes die by FADD/caspase-8-mediated apoptosis (Supplemental Figure 1D). Deficiency in TNFR1 could only partially reduce the spontaneous death of RIPK1-deficient primary hepatocytes, consistent with the partial protective effect of 

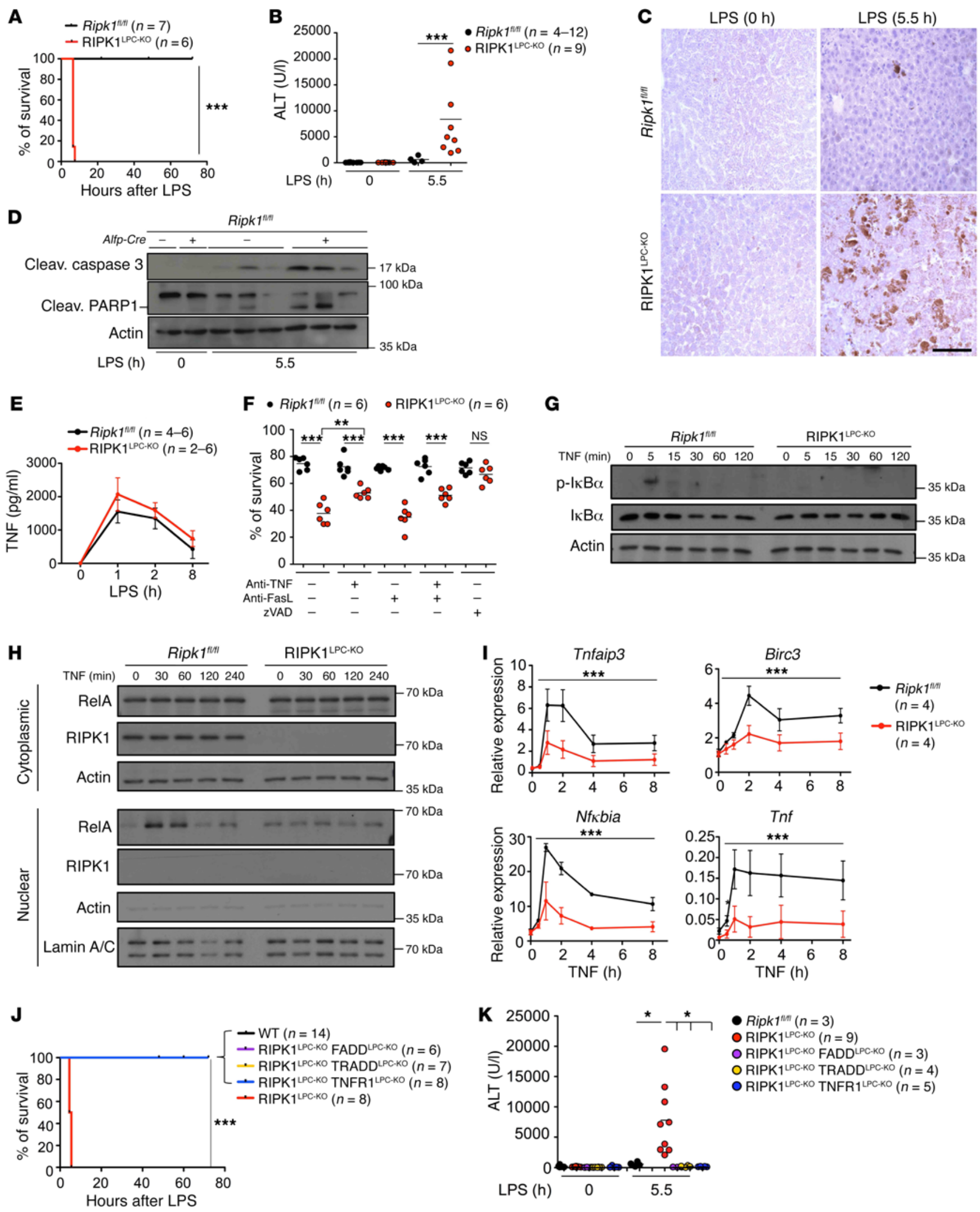

- Ripk1 $1^{\text {nII }}(n=3)$

O RIPK $1^{\text {LPC-KO }}(n=9)$

O RIPK $1^{\text {LPC-KO }}$ FADD ${ }^{\text {LPC-KO }}(n=3)$

O RIPK1 $1^{\text {LPC-KO }}$ TRADD ${ }^{\text {LPC-KO }}(n=4)$

- RIPK $1^{\text {LPC-KO }}$ TNFR1 $1^{\text {LPC-KO }}(n=5)$ 
Figure 1. RIPK1 prevents LPS-induced liver injury by inhibiting TNFmediated hepatocyte death. (A and B) Graphs depicting survival (A) and serum ALT levels (B) of 9-week-old Ripk $7^{f / f l}$ and RIPK1 $1^{\mathrm{LPC}-K 0}$ mice at 5.5 hours after LPS injection. ${ }^{* *} P<0.005$, Mantel-Cox test $(\mathbf{A})$; ${ }^{* * *} P<0.005$, 1-way ANOVA (B). (C) Representative images of liver sections from the indicated mice immunostained for CС3 ( $n=4-5$ per genotype). Scale bar: $100 \mu \mathrm{m}$. (D) Immunoblot analysis for CC3 and cleaved PARP1 in liver lysates from the indicated mice. Actin was used as loading control. (E) Graph depicting serum TNF levels of 9-week-old Ripk $7^{\text {fl/fl }}$ (0 hours, $n=4$; 1 hour, 2 hours, 8 hours, $n=6$ ) and RIPK $1^{\text {LPC-KO }}$ mice (0 hours, 1 hour, 2 hours, $n=6$; 8 hours, $n=2$ ) injected with LPS (mean \pm SEM). (F) Graph depicting

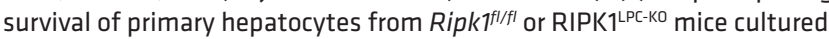
for 24 hours in the presence or absence of anti-TNF, anti-FasL, or Z-VADFMK. ${ }^{* *} P<0.005 ;{ }^{* *} P<0.01$, 1-way ANOVA. (G) Immunoblot analysis of $p-I \kappa B \alpha$ and $I \kappa B \alpha$ in total protein lysates of Z-VAD-FMK-treated Ripk $7^{f \mid / f l}$ and RIPK1 $1^{\mathrm{LPC}-\mathrm{KO}}$ primary hepatocytes stimulated with TNF for the depicted time periods. (H) Immunoblot analysis for RelA in cytoplasmic and nuclear

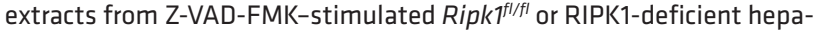
tocytes. Actin and lamin A/C were used as loading controls. (I) qRT-PCR analysis of NF- $\kappa B$ target gene expression in TNF-stimulated Ripk $1^{f l / f l}$ and RIPK ${ }^{\mathrm{LPC}-\mathrm{KO}}$ primary hepatocytes in the presence of Z-VAD-FMK. Mean \pm SEM. ${ }^{* *} P<0.005,2$-way ANOVA. Graphs show relative mRNA expression normalized to Tbp. (J and $\mathbf{K}$ ) Graphs depicting survival (J) and serum ALT levels (K) of 9-week-old mice with indicated genotypes 5.5 hours after LPS

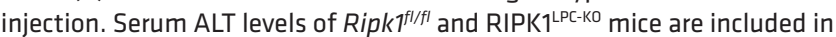
Figure 1B. ${ }^{* * *} P<0.005$, Mantel-Cox test $(\mathrm{J})$; ${ }^{*} P<0.05$, 1-way ANOVA (K).

anti-TNF treatment (Figure 1F and Supplemental Figure 1D). Interestingly, Tradd knockout was almost as efficient as FADD deficiency in protecting RIPK1-deficient hepatocytes from spontaneous death (Supplemental Figure 1D). Therefore, RIPK1-deficient primary hepatocytes undergo spontaneous FADD-caspase-8-dependent apoptosis that is induced in part by TNF and partly by other, yet unknown, receptors that also utilize TRADD as an adapter.

The finding that Fadd knockout could fully protect RIPK1deficient hepatocytes from LPS/TNF-induced death in vivo and in vitro suggested that, in contrast with other cell types in which RIPK1 prevents both apoptosis and necroptosis, in the liver, RIPK1 deficiency sensitizes cells exclusively to apoptosis. To assess the capacity of TNF to trigger necroptosis in hepatocytes, we treated primary hepatocytes from Ripk $1^{f l / f l}$ or RIPK1 $1^{\mathrm{LPC}-\mathrm{KO}}$ mice with TNF in the presence of cycloheximide (CHX) and Z-VAD-FMK. As shown in Supplemental Figure 1E, TNF plus CHX treatment efficiently killed WT hepatocytes and this death was completely prevented in the presence of Z-VAD-FMK. Also, Z-VAD-FMK fully prevented the death of RIPK1-deficient hepatocytes induced by treatment with TNF or TNF plus CHX. Therefore, in contrast with other cell types in which Z-VAD-FMK treatment prevents apoptosis, but sensitizes cells to necroptosis, hepatocytes seem to be resistant to the induction of necroptosis. The very low levels of RIPK3 expression in hepatocytes most likely account for the resistance of these cells to necroptosis (Supplemental Figure 1F).

RIPK1 and RelA cooperate to prevent chronic liver disease and cancer. RIPK $1^{\mathrm{LPC}-\mathrm{KO}}$ mice did not develop signs of spontaneous liver pathology even at the age of 1 year. Moreover, mice with NF-кB deficiency in LPCs do not develop spontaneous liver disease and cancer, in contrast with LPC-specific NEMO-deficient mice, which develop chronic steatohepatitis and HCC (16). We reasoned that RIPK1 may cooperate with NF- $\kappa$ B to inhibit hepatocyte death and prevent chronic liver disease and cancer. To test this hypothesis, we generated mice lacking both RIPK1 and RelA in LPCs (Ripk $1^{f l / f l}$ Rela ${ }^{f / f l}$ Alfp-Cre, hereafter referred to as RIPK1 $1^{\text {LPC-KO }}$ RelA $^{\text {LPC-KO }}$ mice; Figure $\left.2 \mathrm{~A}\right)$. RIPK1 $1^{\text {LPC-KO }}$ RelA $^{\text {LPC-КO }}$ mice showed strongly elevated serum ALT levels, indicating spontaneous liver damage at the age of 8 weeks (Figure 2B). In contrast, ablation of RIPK1 in combination with heterozygous knockout of Rela or ablation of RelA in combination with heterozygous knockout of Ripk1 did not cause considerable spontaneous liver damage (Figure 2B). In addition, about $50 \%$ of the RIPK $1^{\text {LPC-KO }}$ RelA ${ }^{\text {LPC-KO }}$ mice showed mildly elevated total serum bilirubin levels (Figure 2C), which correlated with higher levels of alkaline phosphatase compared with mice with normal bilirubin (Supplemental Figure 2A), indicating the presence of biliary damage. Of note, elevated total bilirubin (BILT) levels correlated with lower ALT levels in these mice (Figure $2 \mathrm{~B}$ ) and reduced ductular response, observed by immunostaining for CK-19 (Supplemental Figure 2B). However, the gross morphology and bile duct integrity were not affected. Immunohistological analysis of liver sections from 8-week-old RIPK1 $1^{\text {LPC-KO }}$ RelA ${ }^{\mathrm{LPC}-\mathrm{KO}}$ mice revealed increased numbers of $\mathrm{CC}^{+}$cells, indicating elevated hepatocyte apoptosis (Figure 2D). In addition, immunostaining for Ki67 revealed strongly elevated numbers of proliferating hepatocytes in the livers of RIPK1 $1^{\mathrm{LPC}-\mathrm{KO}} \mathrm{RelA}^{\mathrm{LPC}-\mathrm{KO}}$ mice (Figure 2D). Moreover, immunostaining for $\mathrm{F} 4 / 80$ revealed increased numbers of macrophages in livers from RIPK1 ${ }^{\mathrm{LPC}-\mathrm{KO}}$ RelA ${ }^{\text {LPC-KO }}$ mice (Supplemental Figure 2C). Furthermore, the livers

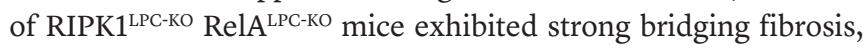
as exemplified by the activation of hepatic stellate cells visualized by immunostaining for $\alpha$-smooth muscle actin ( $\alpha$-SMA) as well as collagen deposition revealed by Sirius red staining (Figure 2D and Supplemental Figure 2C). Therefore, combined ablation of RIPK1 and RelA in LPCs caused spontaneous hepatocyte apoptosis and compensatory proliferation, inflammation, and fibrosis.

Considering that LPS-induced acute liver damage in RIPK1 $1^{\text {LPC-KO }}$ mice depended on TNFR1-mediated hepatocyte apoptosis (see Supplemental Figure 1), we reasoned that the spontaneous development of chronic liver disease in RIPK1 $1^{\text {LPC-KO }}$ RelA $^{\text {LPC-KO }}$ mice might also be driven by TNFR1 signaling. We therefore generated RIPK1 ${ }^{\text {LPC-KO }}$ RelA ${ }^{\text {LPC-KO }}$ mice that additionally lacked TNFR1 in LPCs (Ripk1 $1^{f / f l}$ Rela $^{f l / f l}$ Tnfri $1^{f / f l}$ Alfp-Cre mice, hereafter referred to as $\mathrm{RIPK}^{\mathrm{LPC}-\mathrm{KO}}$ RelA ${ }^{\mathrm{LPC}-\mathrm{KO}}$ TNFR1 ${ }^{\mathrm{LPC}-\mathrm{KO}}$ mice; Figure $\left.2 \mathrm{~A}\right)$. Surprisingly, LPC-specific TNFR1 deficiency did not prevent or ameliorate liver damage and cholestasis in RIPK1 ${ }^{\mathrm{LPC}-\mathrm{KO}} \mathrm{RelA}^{\mathrm{LPC}-\mathrm{KO}}$ mice, as indicated by similar levels of serum ALT (Figure 2B). Lack of TNFR1 did not affect the levels of BILT, but reduced the levels of alkaline phosphatase in the serum of RIPK1 $1^{\text {LPC-KO }}$ RelA ${ }^{\text {LPC-KO }}$ TNFR1 $^{\text {LPC-KO }}$ mice, indicating that TNFR1 signaling contributes to cholangiocyte damage (Supplemental Figure 2A). Moreover, immunohistochemical analysis of liver sections revealed that LPC-specific TNFR1 deficiency did not inhibit spontaneous hepatocyte apoptosis, compensatory proliferation, and fibrosis in RIPK1 $1^{\text {LPC-KO }}$ RelA ${ }^{\text {LPC-KO }}$ mice (Figure 2D). Therefore, in contrast with its essential role in LPS-induced liver damage in RIPK1 $1^{\mathrm{LPC}-\mathrm{KO}}$ mice, TNFR1 signaling in LPCs does not play an important role in the development of spontaneous hepatocyte apoptosis and chronic liver damage caused by combined LPC-specific deficiency of RIPK1 and RelA.

Macroscopic and histological examination of livers from 1 -year-old mice revealed the presence of liver tumors in all 
A

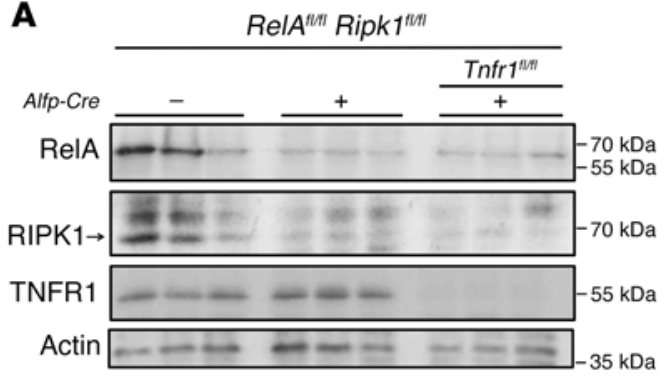

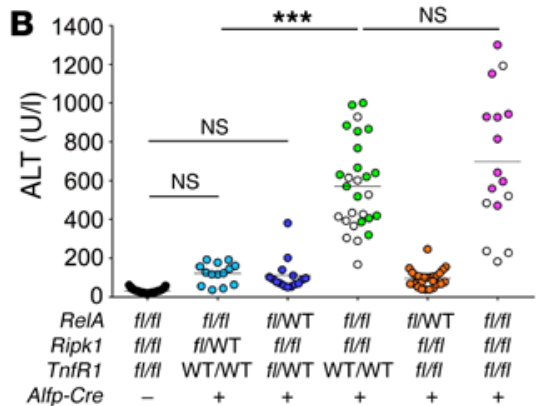

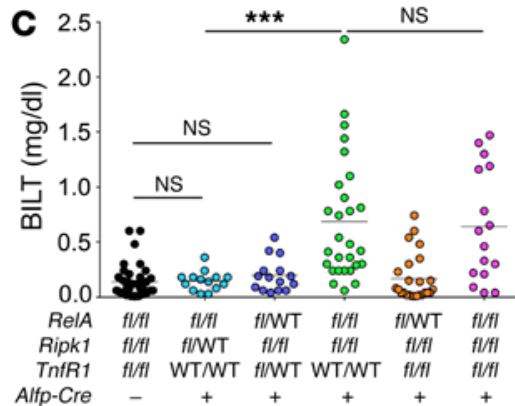

D
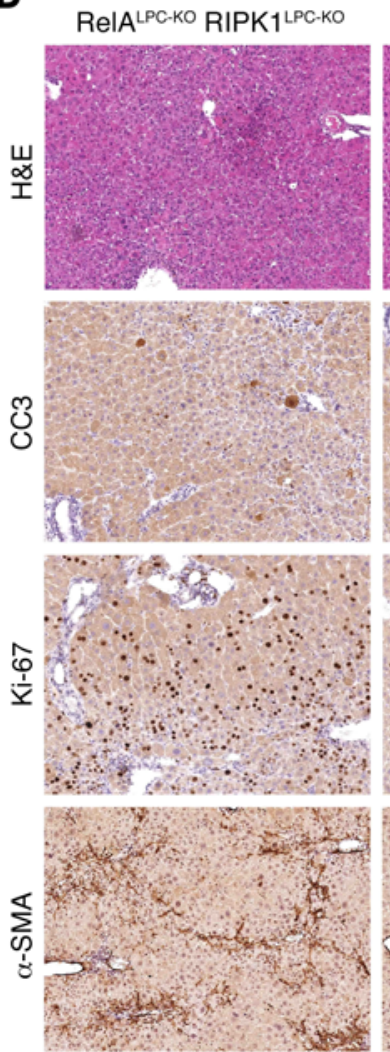

G

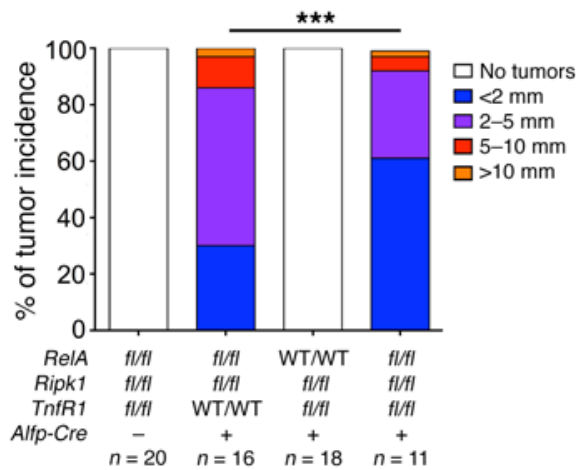

RelA ${ }^{\text {LPC-KO }}$

RIPK1 ${ }^{\text {LPC-KO }}{ }^{\text {TNFR }}$ 1 LPC-KO $^{\text {L }}$
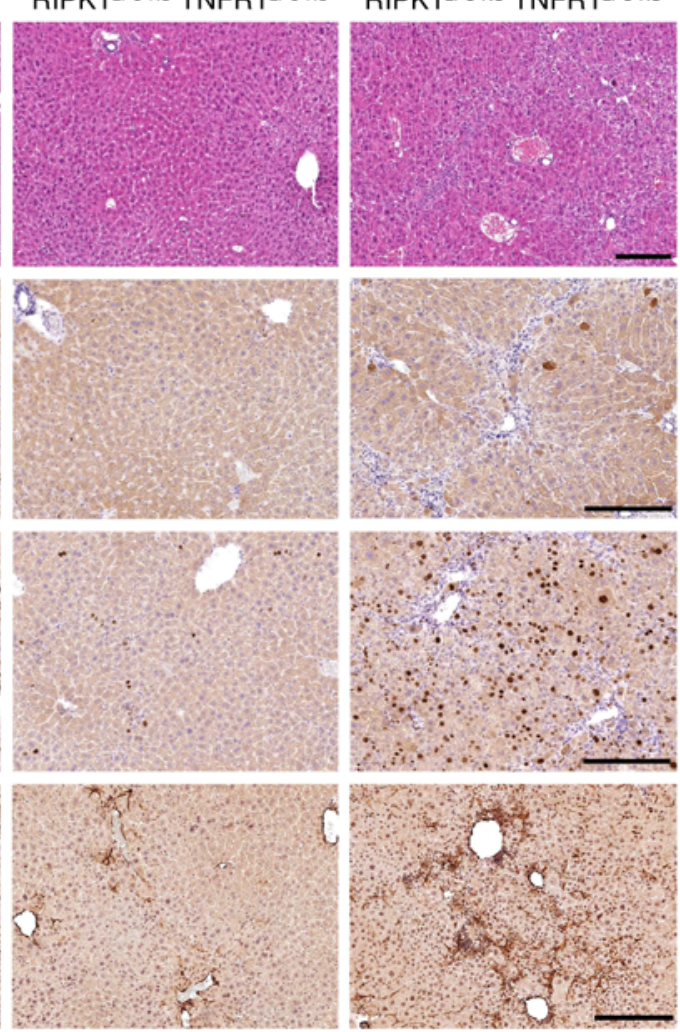

E

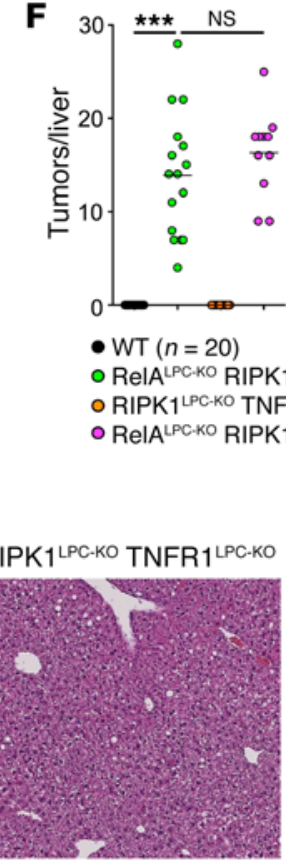

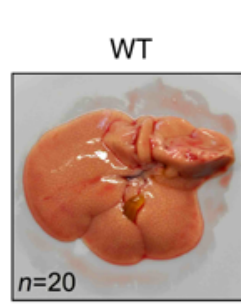

RelA ${ }^{\text {LPC-KO }}$

RIPK1 ${ }^{\text {LPC-KO }}$

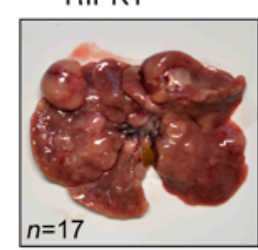

RelA ${ }^{\text {LPC-KO }}$

RIPK1 ${ }^{\text {LPC-KO }}$

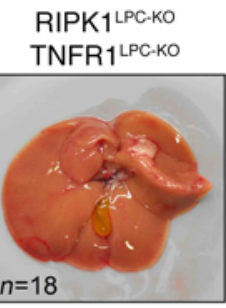

TNFR1 ${ }^{\text {LPC-KO }}$
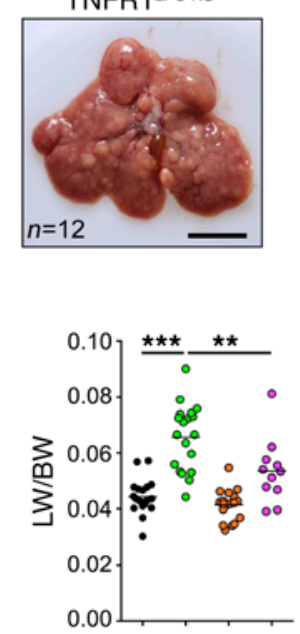

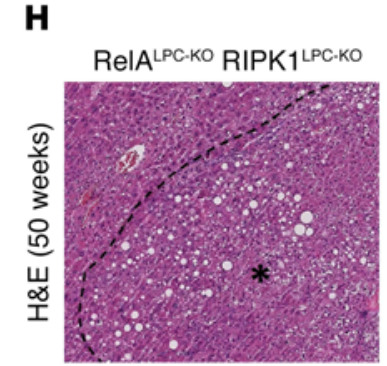

RelA ${ }^{\text {LPC-KO }}$ RIPK1 $1^{\text {LPC-KO }}$ TNFR1 $1^{\text {LPC-KO }}$

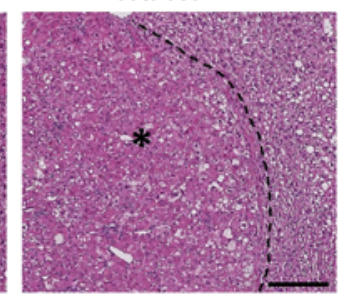

Figure 2. RIPK1 and RelA cooperate to prevent spontaneous hepatocyte death and HCC development independently of TNFR1 signaling. (A) Immunoblot analysis of liver lysates from 8-week-old mice with the indicated genotypes. Actin was used as loading control. (B and C) Graph depicting serum ALT (B) and BILT (C) levels in 8-week-old mice with the indicated genotypes. Empty data points in ALT graph correspond to mice with mildly elevated bilirubin levels ( $>0.7 \mathrm{mg} / \mathrm{dl}$ ). ${ }^{* * *} P<0.005,1$-way ANOVA. (D) Representative images of liver sections from 8-week-old mice with the indicated genotypes are stained with $\mathrm{H} \& \mathrm{E}$ or immunostained with the indicated antibodies. (E) Representative liver images from 50 -week-old mice with the indicated genotypes. (F and G) Tumor load in mice with the indicated genotypes as estimated by quantification of the tumor number per liver and liver weight (LW) to BW ratio (F) and tumor size distribution (C). ${ }^{* * *} P<0.005 ;{ }^{* *} P<0.01,1$-way ANOVA (F); ${ }^{* *} P<0.005, \chi^{2}$ test (C). (H) Representative images of H\&E-stained liver sections from 50-week-old mice with the indicated genotypes. HCC/dysplastic nodule areas are marked with an asterisk. Scale bars: $200 \mu \mathrm{m}$ (D and $\mathbf{H}) ; 1 \mathrm{~cm}(\mathbf{E})$. 
A
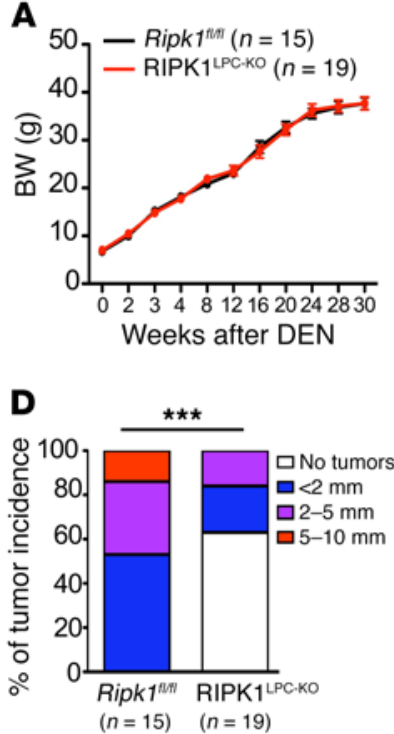

$\mathbf{F}$

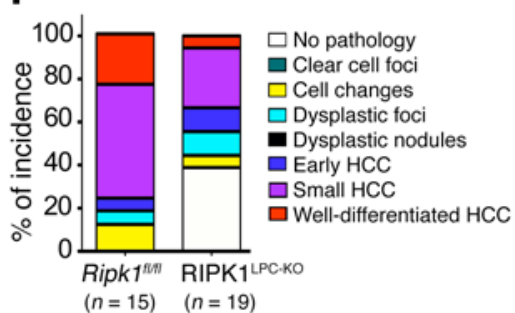

B

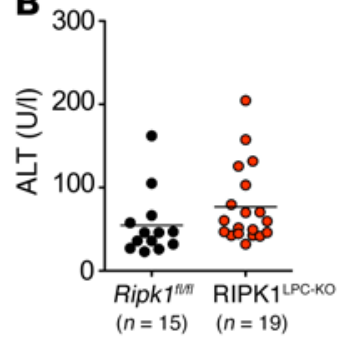

$\mathbf{E}$
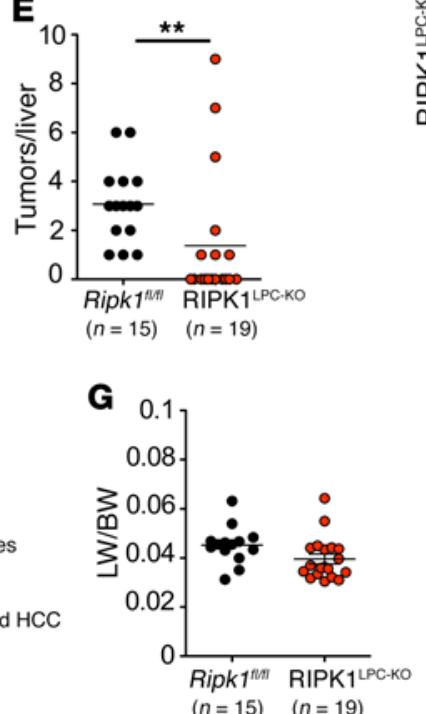
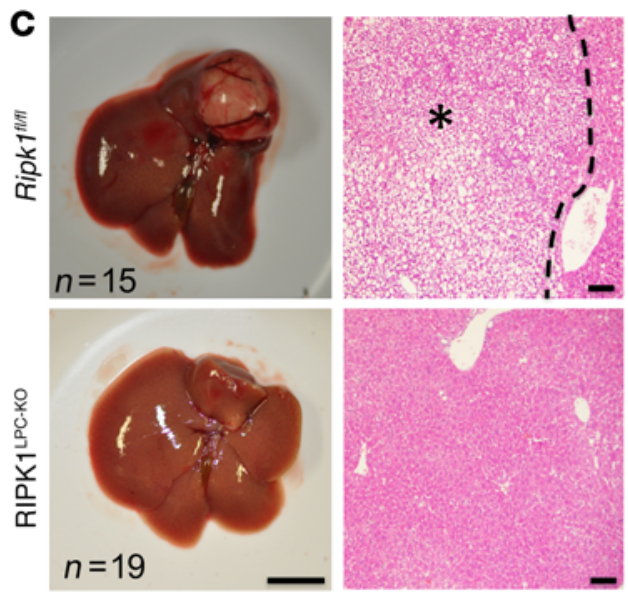
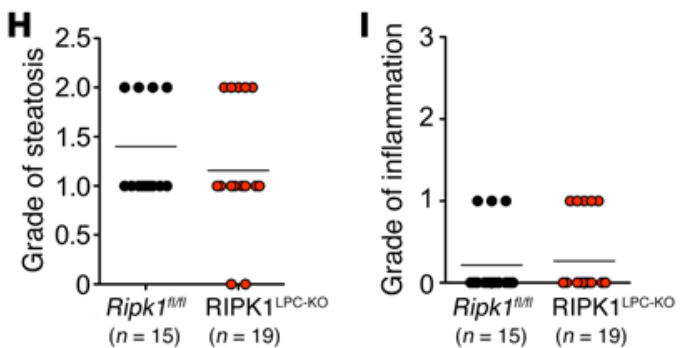

Figure 3. RIPK1 promotes DEN-induced liver tumorigenesis. (A and B) Graphs depicting BW (A) and serum ALT levels at 32 weeks of age (B) of DEN-injected Ripk $7^{f / f f}$ and RIPK1 $1^{\mathrm{LPC}-\mathrm{K} 0}$ mice. Mean \pm SEM (A). (C) Representative photographs of livers and H\&E-stained liver sections from DEN-injected Ripk $7^{f / f f}$ and RIPK 1 ${ }^{\text {LPC-K0 }}$ mice at the age of 32 weeks. Scale bars: $1 \mathrm{~cm}$ (left panels); $100 \mu \mathrm{m}$ (right panels). HCC/dysplastic nodule areas are marked with an asterisk. (D) Tumor load in mice with indicated genotypes as estimated by quantification of tumor size distribution. ${ }^{* *} P<0.005, \chi^{2}$ test. (E) Graphs depicting number of tumors per liver. ${ }^{* *} P<0.01$, Mann-Whitney $U$ test. (F) Histopathological evaluation of HCC development in 32-week-old mice with the indicated genotypes. Each color bar represents the percentage of livers per genotype in which the indicated stage was identified as the most advanced disease stage. (G-I) Liver weight/BW ratio (G), grade of steatosis (H), and grade of inflammation (I) in 32-week-old mice injected with DEN.

RIPK1 ${ }^{\text {LPC-KO }}$ RelA ${ }^{\text {LPC-KO }}$ mice examined (Figures 2, E-H). In contrast, no liver tumors were observed in RIPK1 $1^{\mathrm{LPC}-\mathrm{KO}}$ or RelA ${ }^{\mathrm{LPC}-\mathrm{KO}}$ mice (ref. 16 and data not shown). Thus, the chronic liver disease caused by combined loss of RIPK1 and RelA in LPCs culminated in the development of liver tumors. Consistent with the finding that TNFR1 deficiency did not prevent liver damage in RIPK1 ${ }^{\text {LPC-KO }}$ RelA ${ }^{\text {LPC-KO }}$ mice, 1-year-old RIPK1 ${ }^{\text {LPC-KO }}$ RelA $^{\text {LPC-KO }}$ TNFR1 ${ }^{\text {LPC-KO }}$ mice developed liver tumors (Figure 2, E and F), although their size appeared to be somewhat smaller (Figure $2 \mathrm{G}$ ), suggesting that TNFR1 signaling likely contributes to tumor progression in these mice. Collectively, these results showed that RIPK1- and RelAdependent canonical NF- $\mathrm{BB}$ signaling act synergistically to prevent spontaneously triggered TNFR1-independent hepatocyte apoptosis, chronic liver disease, and the development of liver cancer.

RIPK1 deficiency reduces carcinogen-induced liver tumorigenesis. To study the role of RIPK1 in carcinogen-induced liver tumorigenesis, we used the widely established model of diethylnitrosamineinduced (DEN-induced) liver carcinogenesis (32). Male RIPK1 $1^{\mathrm{LPC}-\mathrm{KO}}$ and Ripk $1^{f l / f l}$ littermate mice were injected with $25 \mathrm{mg} / \mathrm{kg}$ DEN at the age of 2 weeks, and liver tumors were assessed at the age of 32 weeks. RIPK1 $1^{\mathrm{LPC}-\mathrm{KO}}$ and Ripk $1^{t / f l}$ mice showed similar BW gain and serum ALT levels at the age of 32 weeks (Figure 3, A and B), indicating that RIPK1 deficiency did not compromise major liver functions in response to DEN injection. Macroscopic examination revealed liver tumors in all Ripk $1^{f / f f l}$ mice but in less than half of the RIPK1 $1^{\text {LPC-KO }}$ mice, which had fewer and smaller tumors compared with their Ripk1 ${ }^{f / f l}$ littermates (Figure 3, C-E). Histopathological analysis of liver sections confirmed that about $40 \%$ of the RIPK1 $1^{\text {LPC-KO }}$ mice did not show histological signs of liver tumorigenesis. In addition, those RIPK1 $1^{\mathrm{LPC}-\mathrm{KO}}$ mice bearing dysplastic changes in their livers generally showed less advanced tumors (Figure 3F). However, assessment of the liver to BW ratio, liver steatosis, and inflammation as well as apoptosis and proliferation revealed no significant differences between RIPK1 $1^{\mathrm{LPC}-\mathrm{KO}}$ and their Ripk1 $1^{f l f l}$ littermates (Figure 3, G-I, and data not shown). Analysis of liver tumor development 34 weeks after DEN injection confirmed that RIPK1 deficiency inhibited tumorigenesis, as 33\% of the RIPK1 $1^{\text {LPC-KO }}$ mice showed no macroscopically visible tumors, as opposed to $8 \%$ of the control animals (Supplemental Figure 3, A-D). Examination of tumor size and tumor number revealed a similar incidence of large tumors in RIPK1 $1^{\mathrm{LPC}-\mathrm{KO}}$ and Ripk $1^{f / / f l}$ mice at 36 weeks; however, RIPK1 $1^{\mathrm{LPC}-\mathrm{KO}}$ mice had fewer tumors per 
A

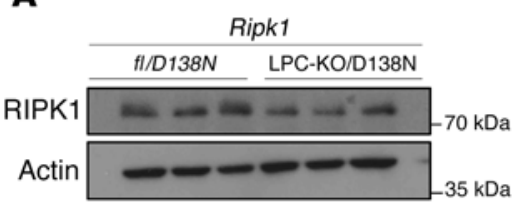

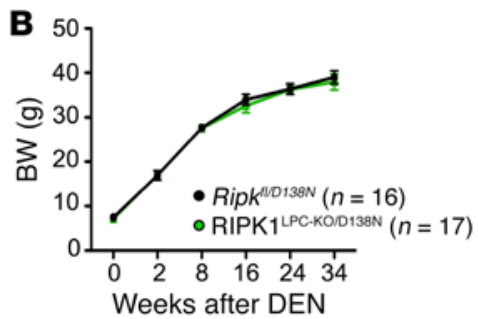
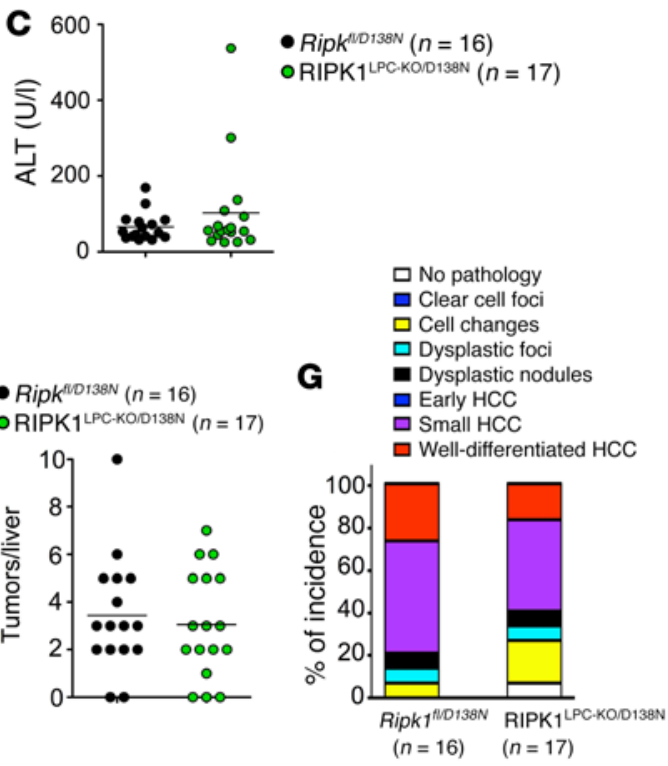

D
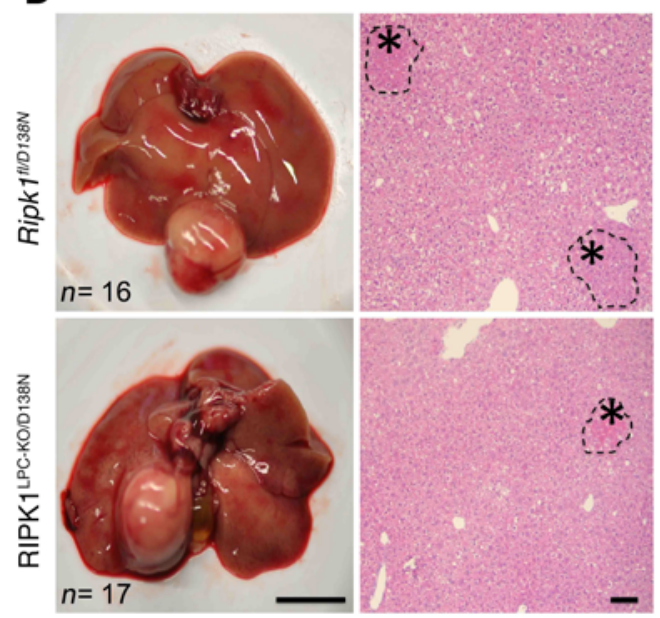

E

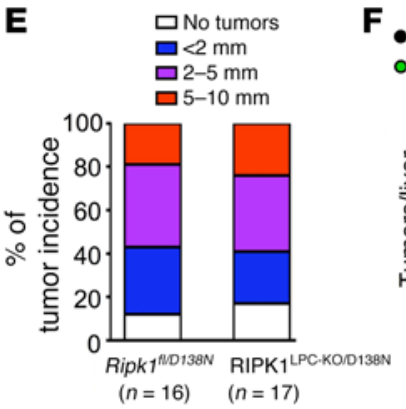
F $\operatorname{Ripk} k^{n D 138 N}(n=16)$ ORIPK1 $1^{\text {LPC-KODD138N }}(n=17)$

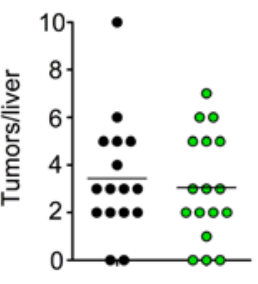

H

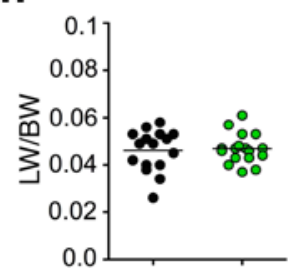

I

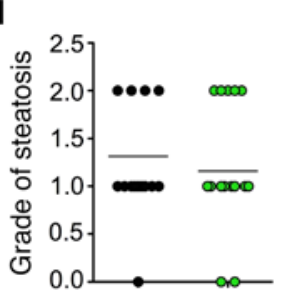

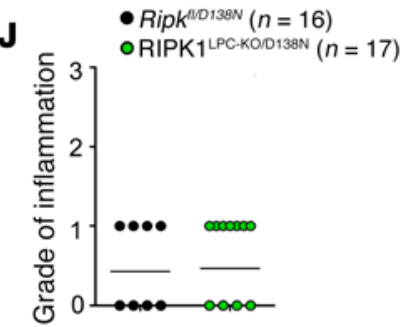

Figure 4. RIPK1 kinase activity does not regulate DEN-induced liver tumorigenesis. (A) Immunoblot analysis for RIPK1 in liver lysates of 9-week-old Ripk $7^{f / / 138 N}$ and RIPK1 $1^{\mathrm{LPC}-K 0 / 0138 \mathrm{~N}}$ mice ( $n=3$ per genotype). (B) BW curve of DEN-injected Ripk $7^{f / D 138 N}$ and RIPK1 $1^{\mathrm{LPC}-\mathrm{KO} / 0138 \mathrm{~N}}$ mice starting from DEN injection until the age of 36 weeks. Mean \pm SEM. (C) Graph depicting serum ALT levels of 36-week-old Ripk $7^{f / D 138 N}$ and RIPK $1^{\text {LPC-ko/D138N }}$ mice upon DEN injection. (D) Representative photographs of livers and H\&E-stained liver sections from DEN-injected Ripk $7^{f / D 138 N}$ and RIPK1 ${ }^{\text {LPC-k0/0138N }}$ mice at the age of 36 weeks. Scale bars: $1 \mathrm{~cm}$ (left panels); $100 \mu \mathrm{m}$ (right panels). HCC/dysplastic nodule areas are marked with an asterisk. (E) Tumor load in 36-week-old Ripk $7^{f / 0138 \mathrm{~N}}$ and RIPK1 $1^{\mathrm{PC}-\mathrm{Ko} / \mathrm{D} 138 \mathrm{~N}}$ mice as estimated by quantification of the tumor size distribution. (F) Graphs depicting the number of tumors per liver. (C) Histopathologi-

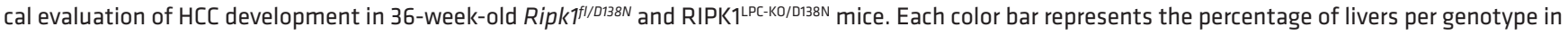
which the indicated stage was identified as the most advanced disease stage ( $n=16-17$ per genotype). (H-J) Liver weight/BW ratio (H), grade of steatosis (I), and grade of inflammation (J) in 36-week-old mice injected with DEN.

liver compared with Ripk1 $1^{f / f l}$ littermates (Supplemental Figure 3, $\mathrm{C}-\mathrm{E}$ ). Taken together, these results showed that RIPK1 deficiency reduced $\mathrm{DEN}$-induced liver tumorigenesis by decreasing overall tumor incidence and tumor numbers, but also by slowing down tumor progression.

RIPK1 kinase activity does not regulate DEN-induced liver tumorigenesis. To address the role of RIPK1 kinase activity in DENinduced liver tumorigenesis, we employed the Ripk1 $1^{D 138 N / D 138 N}$ knockin mice expressing catalytically inactive RIPK1 D138N from the endogenous Ripk1 genomic locus (33). To investigate the LPCspecific function of RIPK1 kinase activity, we employed mice that carry 1 loxP-flanked and 1 RIPK1 D138N mutant allele crossed to Alfp-Cre transgenic mice (Ripk1 $1^{f / D 138 N} A l f p-C r e^{T g / W T}$, hereafter referred to as RIPK1 $1^{\mathrm{LPC}-\mathrm{KO} / \mathrm{D} 138 \mathrm{~N}}$ mice). In these mice, Cre-mediated recombination deletes the loxP-flanked Ripk1 allele in LPCs; therefore, these cells express only the kinase-inactive RIPK1 D138N protein (Figure $4 \mathrm{~A}$ ). Male RIPK1 ${ }^{\mathrm{LPC}-\mathrm{KO} / \mathrm{D} 138 \mathrm{~N}}$ and Ripk1 $1^{f / D 138 N}$ littermates were injected with $25 \mathrm{mg} / \mathrm{kg}$ DEN at the age of 2 weeks, and liver tumors were assessed at the age of 36 weeks. Ripk $1^{f / D 138 N}$ and
RIPK1 $1^{\mathrm{LPC}-\mathrm{KO} / \mathrm{D} 138 \mathrm{~N}}$ mice did not show differences in $\mathrm{BW}$ gain and serum ALT levels (Figure 4, B and C). Macroscopic liver examination revealed similar tumor incidence, tumor numbers, and also tumor size in RIPK1 $1^{\mathrm{LPC}-\mathrm{KO} / \mathrm{D} 138 \mathrm{~N}}$ mice compared with their control littermates (Figure 4, D-F). Histopathological examination of liver sections revealed an overall similar incidence of advanced and early tumors in RIPK1 $1^{\mathrm{LPC}-\mathrm{KO} / \mathrm{D} 138 \mathrm{~N}}$ and Ripk1 $1^{f / \mathrm{D} 138 N}$ mice (Figure 4, G-J). Therefore, lack of RIPK1 kinase activity did not inhibit DENinduced liver tumorigenesis, suggesting that RIPK1 promotes DEN-induced liver tumor development via kinase-independent scaffolding functions.

RIPK1 prevents DEN-induced hepatocyte death. To investigate the RIPK1-dependent mechanisms regulating DEN-induced tumorigenesis, we examined the role of RIPK1 in hepatocyte responses to DEN-induced DNA damage. Measurement of serum ALT levels revealed increased liver damage in RIPK1 $1^{\mathrm{LPC}-\mathrm{KO}}$ compared with Ripk $1^{f / f l}$ littermates at 6 and 24 hours after DEN administration (Figure 5A). Immunohistochemical analysis of CC3 revealed increased numbers of apoptotic cells in the liver 

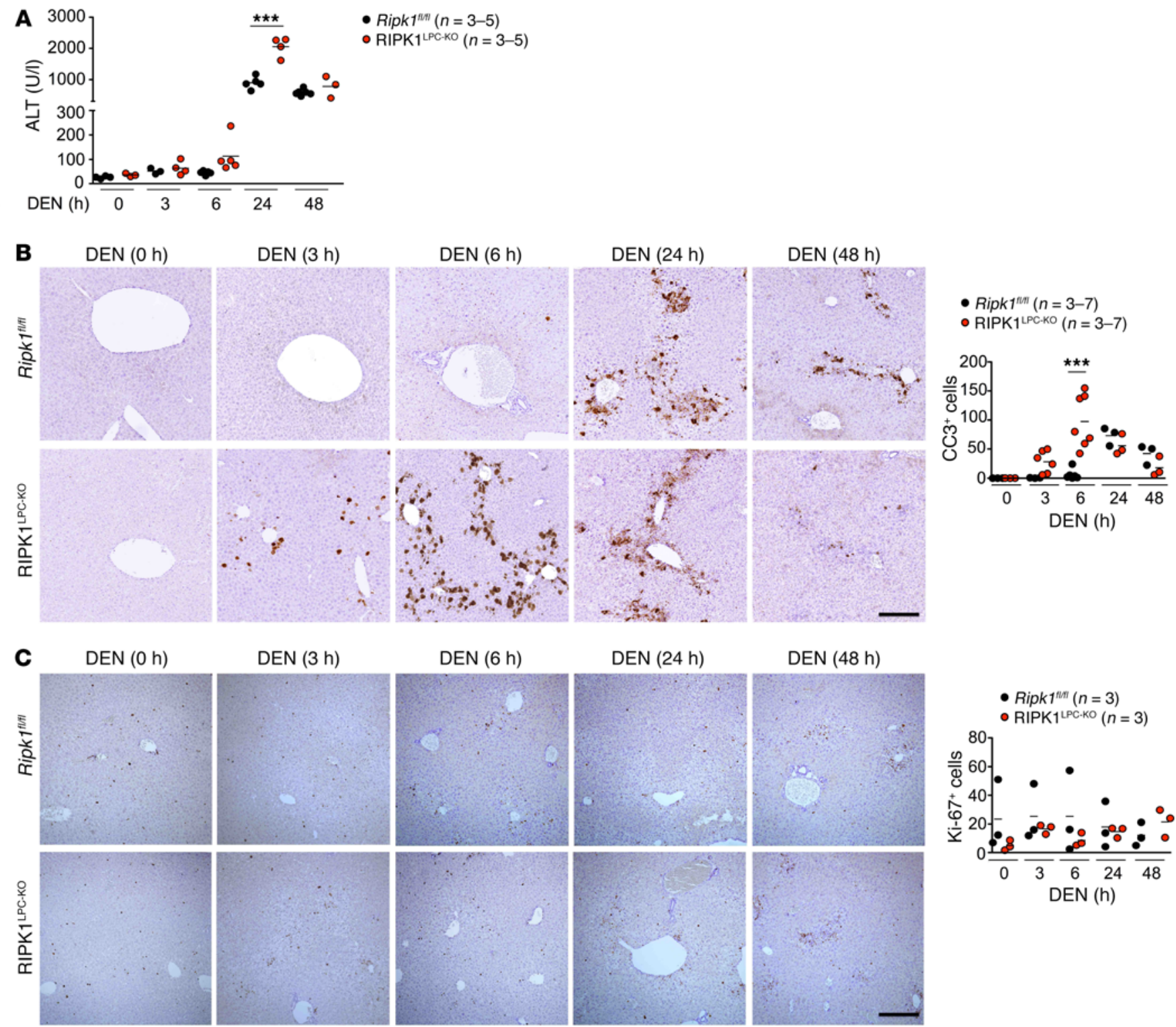

D $\quad \mathrm{DEN}(\mathrm{Oh})$
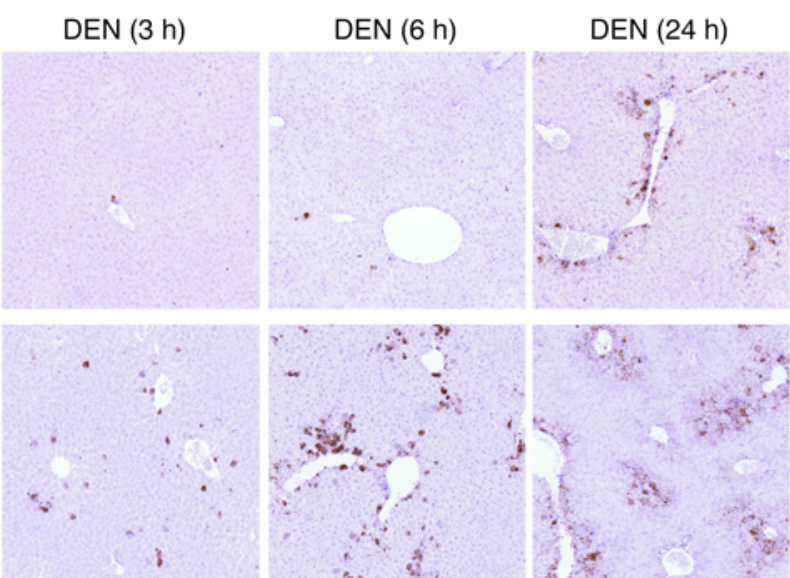

\section{DEN (48 h)}
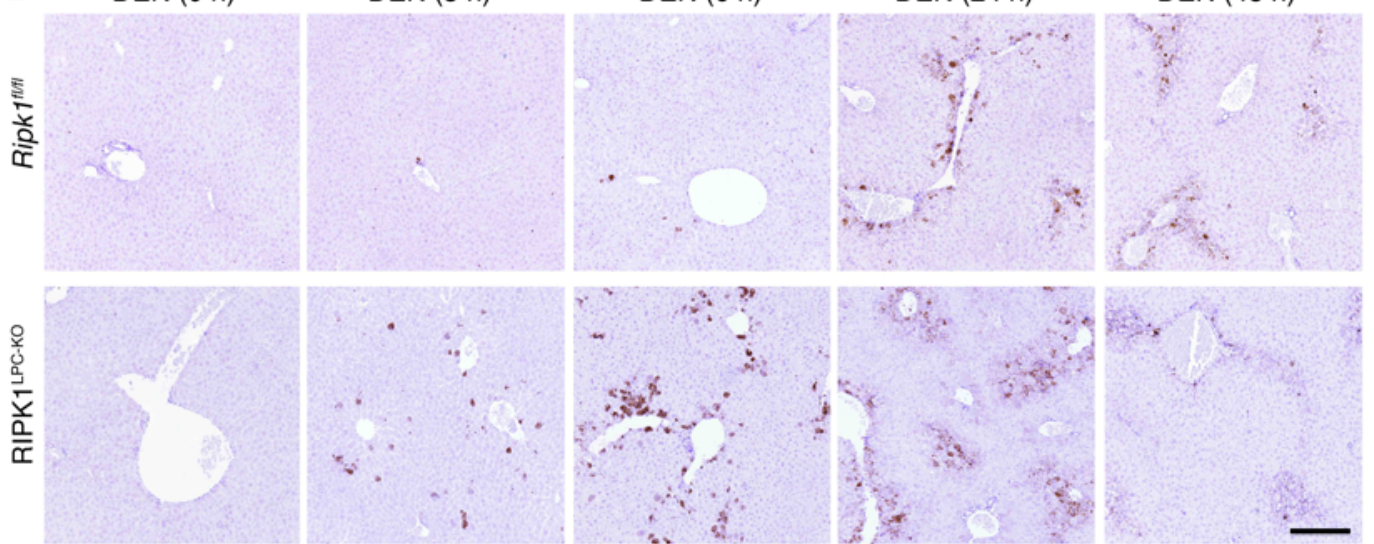

- $\operatorname{Ripk}^{\text {nim }}(n=3-7)$

- RIPK1 $1^{\text {LPC-KO }}(n=3-7)$

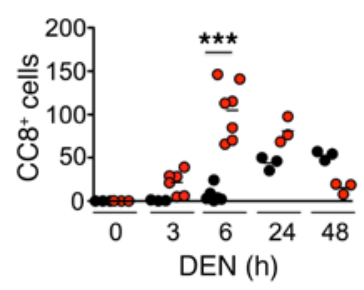

Figure 5. RIPK1 regulates DEN-induced apoptotic death of hepatocytes. (A) Graph depicting serum ALT levels of 6-week-old Ripk $7^{\text {fl/fl }}$ and RIPK1 ${ }^{\mathrm{LPC}-\mathrm{k} 0}$ mice injected with an acute dose of DEN for the indicated time periods. ${ }^{* *} P<0.005,1$-way ANOVA. (B and $\left.\mathbf{D}\right)$ Representative images of livers of noninjected or DEN-injected (acute dose) 6-week-old Ripk $7^{f / f f l}$ and RIPK $1^{\text {PC-Ko }}$ mice stained for CC3 (B), Ki-67 (C), and CC8 (D). Scale bars: $100 \mu \mathrm{m}$. Quantification of CC3 ${ }^{+}$ $\mathrm{Ki}-67^{+}$, and $\mathrm{CC} 8^{+}$cells per field, mean of 5 fields per animal. ${ }^{* * *} P<0.005,1$-way ANOVA. 

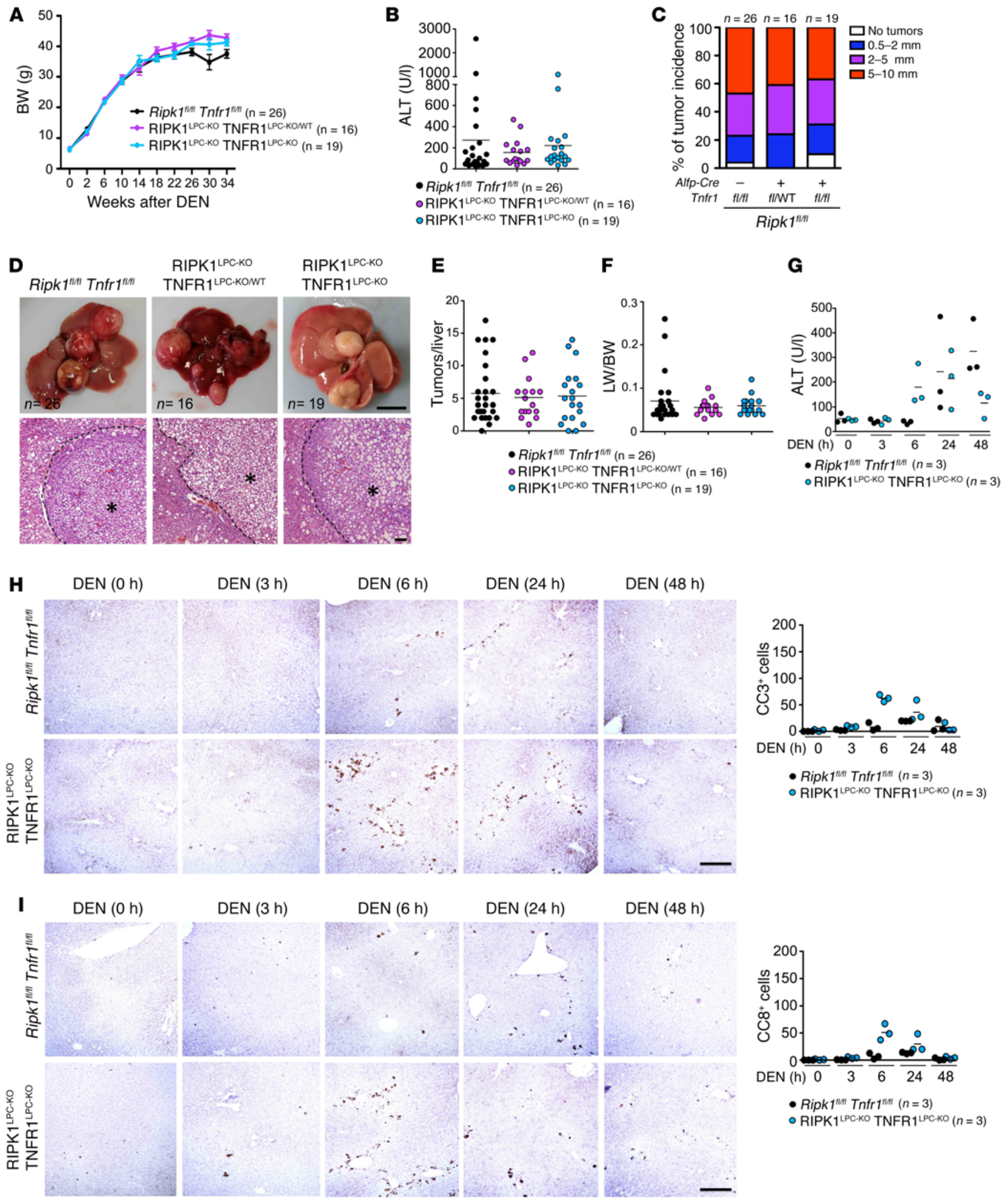
Figure 6. TNFR1 signaling inhibits DEN-induced liver tumorigenesis in RIPK1 $1^{\text {LPC-Ko }}$ mice. (A) BW curve of DEN-injected Ripk $7^{f l / f l} \mathrm{Tnfr} 7^{f / f f l}$, RIPK1 $1^{\mathrm{LPC}-\mathrm{K} 0}$ TNFR1 $1^{\text {LPC-KO/WT }}$, and RIPK1 ${ }^{\text {LPC-KO }}$ TNFR $1^{\text {LPC-KO }}$ mice starting from DEN injection until the age of 36 weeks. Mean \pm SEM. (B) Graph depicting serum

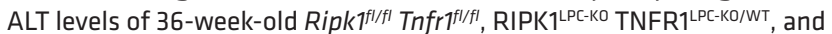
RIPK1 ${ }^{\mathrm{LPC}-K 0}$ TNFR1 ${ }^{\mathrm{LPC}-K 0}$ mice upon DEN injection. (C) Tumor load in 36-weekold Ripk $1^{f / / f l}$ Tnfr $1^{f / / f 1}$, RIPK1 $1^{\text {LPC-Ko }}$ TNFR1 $1^{\text {LPC-KO/WT }}$, and RIPK1 ${ }^{\text {LPC-Ko }}$ TNFR1 $1^{\text {LPC-Ko }}$ mice as estimated by quantification of the tumor size distribution. (D) Representative photographs of livers and H\&E-stained liver sections from DEN-injected Ripk fl/fl $^{\text {Tnfr }}$ Tr $^{f / f f l}$, RIPK $1^{\text {LPC-KO }}$ TNFR1 $1^{\text {LPC-KO/WT }}$, and RIPK1 ${ }^{\text {LPC-KO }}$ TNFR $1^{\mathrm{PPC}-\mathrm{KO}}$ mice at the age of 36 weeks. Scale bars: $1 \mathrm{~cm}$ (upper panels); $100 \mu \mathrm{m}$ (lower panels). HCC/dysplastic nodule areas are marked with an asterisk. (E and $\mathbf{F}$ ) Graphs depicting the number of tumors per liver (E) and LW/BW ratio (F) in 36-week-old mice injected with DEN. (G) Graph depicting serum ALT levels of 6-week-old Ripk fl/fl $^{f n} \mathrm{Fr}^{\mathrm{fl} / f l}$ and RIPK1 $1^{\mathrm{LPC}-\mathrm{Ko}}$ $\mathrm{TNFR}^{\mathrm{LPC}-\mathrm{KO} 0}$ mice injected with an acute dose of DEN for the indicated time periods. (H and I) Representative images of livers of noninjected or DENinjected (acute dose) 6-week-old Ripk $7^{f / f l} \mathrm{Tnfr}^{\mathrm{fl} / f \mid}$ and RIPK1 $1^{\mathrm{LPC}-\mathrm{Ko}} \mathrm{TNFR}^{\mathrm{LPC}-\mathrm{KO}}$ mice stained for CC3 (H) and CC8 (I). Scale bars: $100 \mu \mathrm{m}$. Quantification of CC3 and CC8 cells per field (mean of 5 fields per animal).

of RIPK1 $1^{\text {LPC-KO }}$ mice compared with Ripk1 $1^{\text {flf }}$ littermates at 3 and 6 hours after DEN injection, showing that RIPK1 deficiency sensitized hepatocytes to DEN-induced apoptosis (Figure 5B). Earlier studies suggested that increased DEN-induced apoptosis of IKK2deficient hepatocytes caused increased compensatory proliferation, resulting in enhanced DEN-induced tumorigenesis in mice with hepatocyte-specific IKK2 deficiency (34). Immunostaining for the cell proliferation marker Ki67 did not show increased proliferation in the liver of RIPK $1^{\text {LPC-KO }}$ compared with Ripk $1^{f / f / l}$ mice in response to DEN injection (Figure 5C), suggesting that increased hepatocyte apoptosis did not cause increased compensatory proliferation in this model.

Immunostaining of liver sections with antibodies recognizing phosphorylated histone $\mathrm{H} 2 \mathrm{AX}(\gamma \mathrm{H} 2 \mathrm{AX})$, which is generated in the vicinity of DNA damage lesions (35), revealed comparable numbers of $\gamma \mathrm{H} 2 \mathrm{AX}^{+}$hepatocytes in RIPK1 $1^{\mathrm{LPC}-\mathrm{KO}}$ and Ripk $1^{f / f l}$ littermates (Supplemental Figure 4, A and B). DNA damage induces the stabilization and activation of the tumor suppressor p53, which maintains genomic stability by controlling the expression of genes regulating cellular senescence, cell-cycle progression, cell death, and DNA repair (36). Assessment of p53 activation by immunoblot analysis of liver protein extracts and quantitative reverse-transcription PCR (qRT-PCR) analysis of the mRNA expression of key p53 target genes did not reveal considerable differences between RIPK1 ${ }^{\mathrm{LPC}-\mathrm{KO}}$ and Ripk1 $1^{\ell / l}$ mice (Supplemental Figure 4, C and D). These results suggest that the increased hepatocyte apoptosis in DEN-injected RIPK1 $1^{\mathrm{LPC}-\mathrm{KO}}$ mice is not caused by increased DNA damage-induced p53 activation.

To assess whether death receptor signaling contributes to the increased hepatocyte apoptosis in DEN-injected RIPK1 ${ }^{\mathrm{LPC}-\mathrm{KO}}$ mice, we stained liver sections with antibodies recognizing CC8. Indeed, RIPK1 ${ }^{\mathrm{LPC}-\mathrm{KO}}$ mice showed increased numbers of $\mathrm{CC} 8^{+}$hepatocytes at 3 and 6 hours after DEN administration (Figure 5D), suggesting that RIPK1-deficient hepatocytes were sensitized to DEN-induced cell death driven primarily by death receptor-induced caspase-8-dependent apoptosis. Gene expression analysis revealed the upregulation of Tnf and Trail in the liver of RIPK1 ${ }^{\mathrm{LPC}-\mathrm{KO}}$ and Ripk $1^{f / f l}$ littermates within 3 hours after DEN injection (Supple- mental Figure 5), suggesting that they could trigger apoptosis of RIPK1-deficient hepatocytes.

To address the role of RIPK1 kinase activity, we compared DENinduced hepatocyte responses in Ripk1 $1^{f / D 138 N}$ and RIPK1 $1^{L P C-K O / D 138 N}$ mice. In contrast with RIPK1 1 LPC-Ko, RIPK1 $1^{\text {LPC-KO/D138N }}$ mice did not show considerably increased serum ALT levels compared with their Ripk1 $1^{7 / D 138 N}$ littermates in response to DEN injection (Supplemental Figure 6A). Moreover, RIPK1 $1^{\mathrm{LPC}-\mathrm{KO} / \mathrm{D} 138 \mathrm{~N}}$ mice did not show increased CC3- or CC8-positive cells in their livers compared with Ripk1 $1^{f / D 138 N}$ control mice (Supplemental Figure 6, B and C). Therefore, lack of RIPK1 kinase activity did not sensitize hepatocytes to DEN-induced liver damage, showing that kinase-independent RIPK1 scaffolding functions protect hepatocytes from apoptosis in response to DEN injection in vivo.

TNFR1 deficiency restores DEN-induced tumorigenesis in RIPK1 ${ }^{\text {LPC-KO }}$ mice. To address whether TNFR1-mediated hepatocyte apoptosis contributes to the reduced DEN-induced tumorigenesis in RIPK1 ${ }^{\mathrm{LPC}-\mathrm{KO}}$ mice, we generated mice lacking both RIPK1 and TNFR1 in LPCs (RIPK1 ${ }^{\text {LPC-KO }}$ TNFR1 $1^{\text {LPC-KO})}$ by crossing Ripk1 $1^{f f l}$ Alfp-Cre ${ }^{T_{g} / W T}$ to Tnfr $1^{f / f l}$ mice. RIPK1 ${ }^{\text {LPC-KO }}$ TNFR1 $1^{\text {LPC-KO }}$ mice and their floxed littermates injected with $25 \mathrm{mg} / \mathrm{kg}$ DEN at the age of 2 weeks did not show differences in BW and serum ALT levels (Figure 6, A and B). Moreover, macroscopic assessment of livers from mice sacrificed at 36 weeks revealed similar incidence, numbers, and size of tumors in RIPK1 $1^{\text {LPC-KO }}$ TNFR $1^{\text {LPC-KO }}$ and RIP-

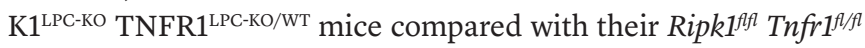
littermates (Figure 6, C-F). Therefore, heterozygous or homozygous Tnfr 1 knockout in LPCs restored DEN-induced liver tumorigenesis in RIPK1 ${ }^{\text {LPC-KO }}$ mice, suggesting that the protective effect of RIPK1 deficiency depends on TNFR1 signaling in LPCs.

Since RIPK1 deficiency sensitizes hepatocytes to TNF-induced apoptosis, we assessed whether Tnfrl knockout could prevent DEN-induced hepatocyte apoptosis in the liver of RIPK1 $1^{\text {LPC-KO }}$ mice. RIPK1 $1^{\text {LPC-KO }}$ TNFR1 $1^{\text {LPC-KO }}$ mice did not show increased hepatocyte apoptosis at 3 hours after DEN injection, although they had increased numbers of apoptotic hepatocytes and elevated serum ALT levels at 6 hours after DEN injection (Figure 6, G-I). Therefore, TNFR1 deficiency prevented the early hepatocyte apoptosis observed in RIPK1 $1^{\mathrm{LPC}-\mathrm{KO}}$ mice at 3 hours after DEN injection, suggesting that TNFR1-mediated death of hepatocytes may at least in part be responsible for the reduced tumor development in these mice, perhaps by eliminating cells carrying DNA damage.

RIPK1 ameliorates obesity-induced liver carcinogenesis. Genetic or high-fat diet-induced (HFD-induced) obesity exacerbates DEN-induced liver carcinogenesis in a TNFR1-dependent manner $(37,38)$. To address the role of RIPK1 in obesity-associated liver carcinogenesis, we injected RIPK1 $1^{\mathrm{LPC}-\mathrm{KO}}$ and Ripk $1^{\mathrm{fl} / \mathrm{l}}$ male littermates with DEN and subsequently divided them into 2 groups, one receiving HFD starting from the age of 4 weeks and a control group receiving normal chow diet (NCD). HFD-fed RIPK1 1PC-KO mice showed similar BW gain, serum LDL cholesterol, and ALT values compared with HFD-fed Ripk1 $1^{f / f l}$ littermates (Figure 7, $\mathrm{A}-\mathrm{C})$. In addition, RIPK1 ${ }^{\mathrm{LPC}-\mathrm{KO}}$ mice showed similar responses compared with their Ripk1 $1^{t / f l}$ littermates in glucose tolerance tests performed at 12 and 24 weeks of age (Figure 7D). Therefore, hepatic RIPK1 deficiency does not affect the development of HFD-induced obesity and glucose intolerance. Macroscopic 
A

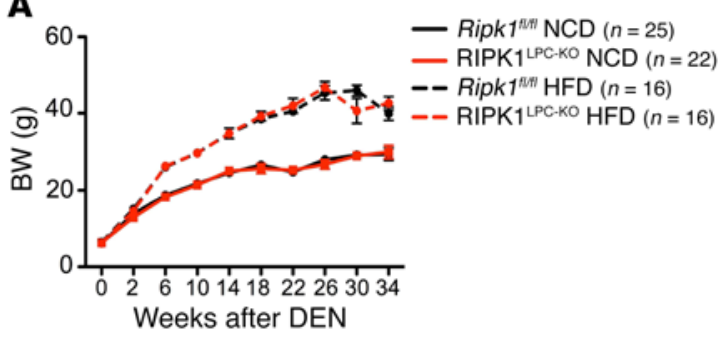

B

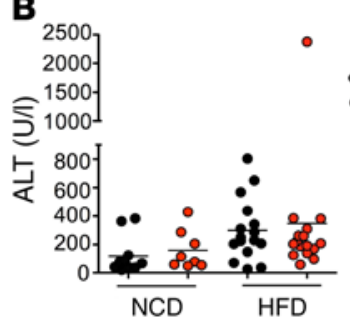

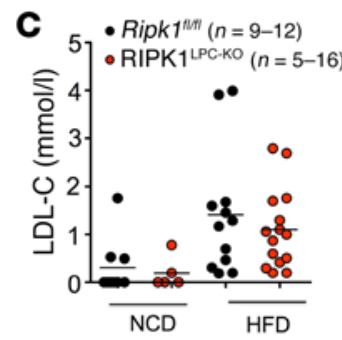

D
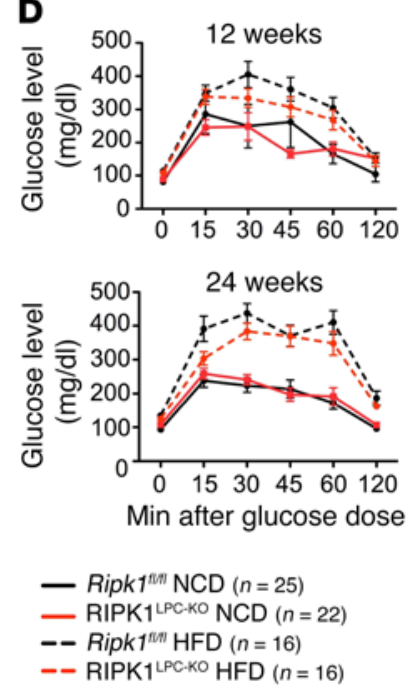

E

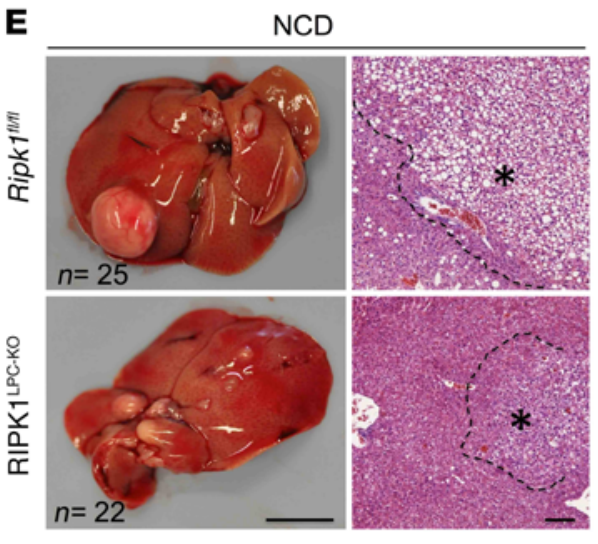

HFD

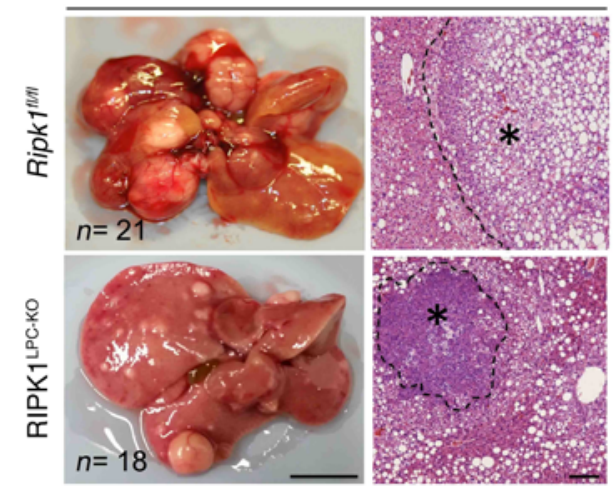

$\mathbf{F}$

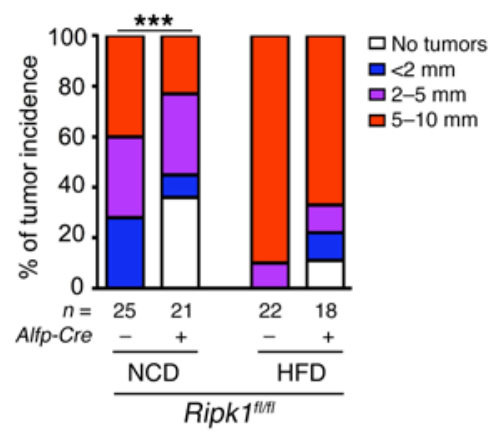

G $\quad-\operatorname{Ripk}^{\text {nal }}(n=21-25)$
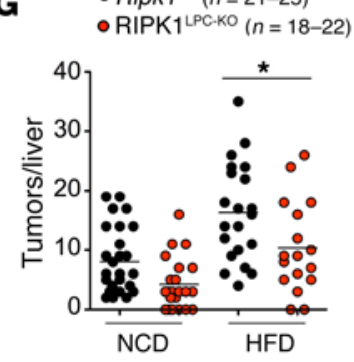

Figure 7. LPC-specific deficiency of RIPK1 attenuates obesity-induced liver carcinogenesis. (A) BW curve of NCD- or HFD-fed DEN-injected Ripk fl/fl $^{\text {and }}$ RIPK1 ${ }^{L P C-K O}$ mice starting from DEN injection until the age of 36 weeks. Mean \pm SEM. (B and C) Graph depicting serum ALT level (B) and serum LDL cholesterol level (LDL-C) (C) of NCD- or HFD-fed 36-week-old Ripk $7^{\text {fl/fl }}$ and RIPK1 ${ }^{\text {LPC-Ko }}$ mice upon DEN injection. (D) Graphs depicting blood glucose levels after glucose administration in NCD- or HFD-fed Ripk fl/fl $^{f / 2}$ and RIPK $1^{\mathrm{LPC}-\mathrm{KO}}$ mice at 12 and 24 weeks of age. Mean $\pm \mathrm{SEM}$. (E) Representative photographs of livers and H\&E-stained liver sections from DEN-injected Ripk fl/fl $^{f / 2}$ and RIPK $1^{\text {LPC-Ko }}$ mice at the age of 36 weeks. Scale bars: $1 \mathrm{~cm}$ (left panels); $100 \mu \mathrm{m}$; (right panels). HCC/dysplastic nodule areas are marked with an asterisk. (F) Tumor load in NCD- or HFD-fed 36-week-old Ripk $7^{f / f l}$ and RIPK $1^{\text {LPC-KO }}$ mice as estimated by quantification of the tumor size distribution. ${ }^{* * *} P<0.005, \chi^{2}$ test. (G) Graphs depicting the number of tumors per liver in 36-week-old mice injected with DEN. ${ }^{*} P<0.05,1$-way ANOVA.

assessment of liver tumors 34 weeks after DEN injection in mice fed with HFD revealed reduced tumor incidence, but also tumor number and size, in RIPK1 $1^{\mathrm{LPC}-\mathrm{KO}}$ mice compared with Ripk1 $1^{f / f l}$ littermates, although the protective effect of RIPK1 deficiency was not as pronounced as in mice fed with NCD (Figure 7, E-G). Therefore, RIPK1 also contributes to the increased DEN-induced tumorigenesis caused by diet-associated obesity.

\section{Discussion}

In this study, we have addressed the role of RIPK1 kinase activity-dependent and -independent functions in liver homeostasis, hepatocyte death, and liver injury as well as in hepatocarcino- genesis. Our findings underscore important RIPK1 functions in regulating TNFR1-dependent and -independent hepatocyte survival and liver tumorigenesis. We found that RIPK1 prevents endotoxin-induced liver damage by inhibiting TNFR1-induced hepatocyte death. These results are in agreement with recent reports showing that RIPK1-deficient hepatocytes were sensitive to TNF-induced death and that mice with LPC-specific Ripk1 knockout were highly susceptible to ConA- or LPS-induced liver damage (20-22, 24). Our genetic studies showed that TNF induces hepatocyte death in RIPK1-deficient hepatocytes by activating TNFR1-TRADD-FADD-dependent apoptosis both in vivo and in vitro (Figure 8A). The essential role of TRADD in TNFR1-induced 
A

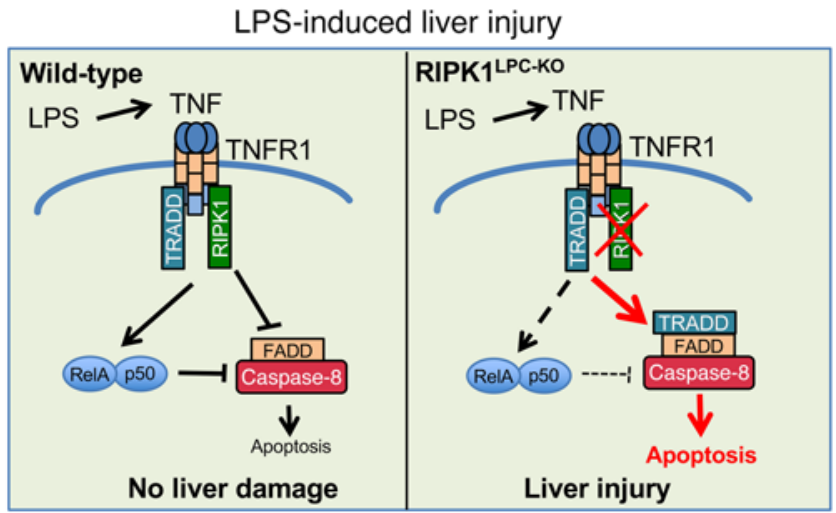

B

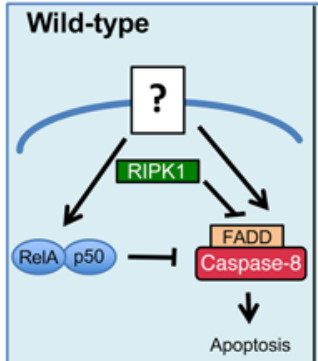

Healthy liver
Steady state liver homeostasis

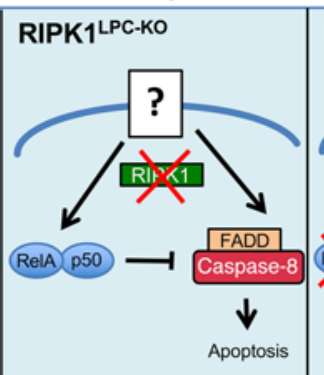

Healthy liver

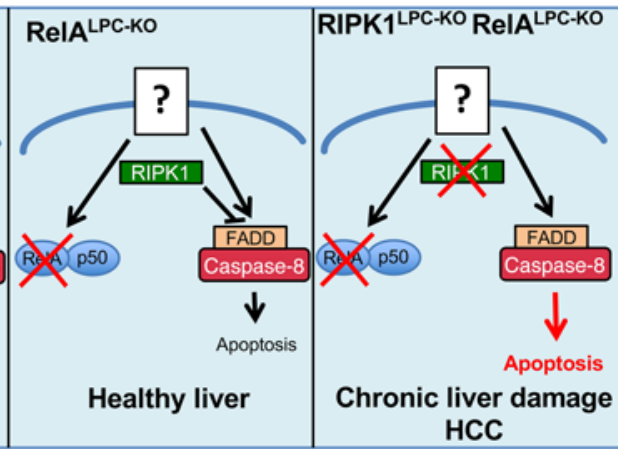

DEN-induced tumorigenesis

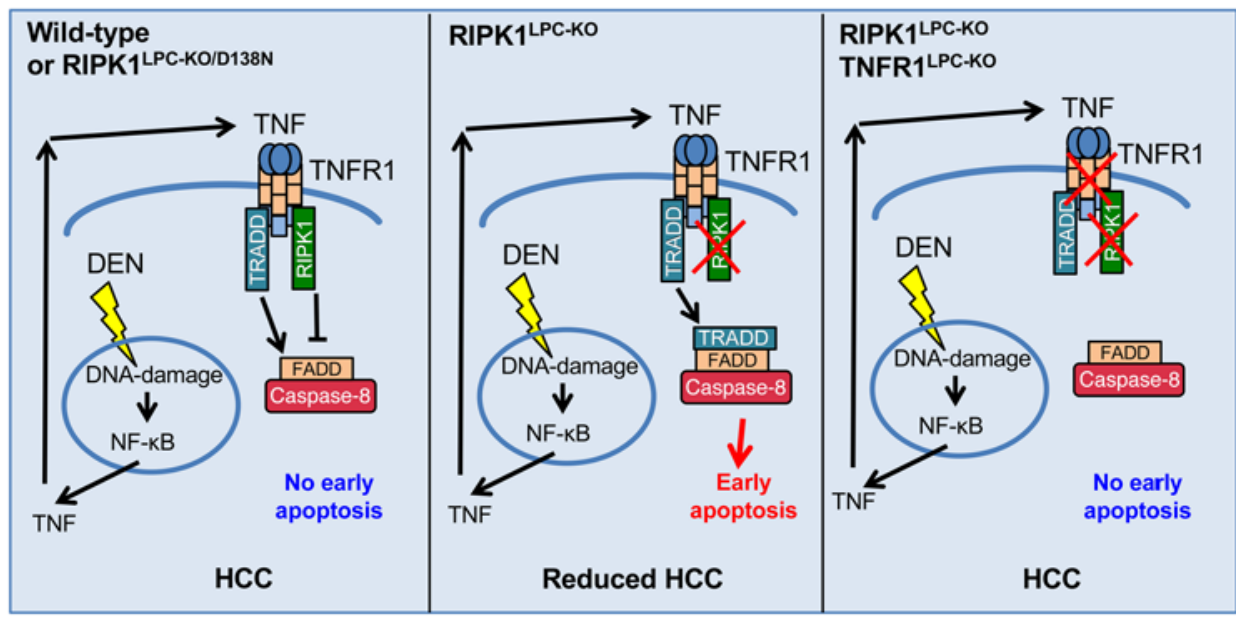

\section{C}

Figure 8. Schematic depictions of the different functions of RIPK1 in the liver.

(A) RIPK1 prevents LPS-induced liver injury by protecting hepatocytes from TNF-induced apoptosis. In RIPK1 $1^{\mathrm{LPC}-\mathrm{KO}}$ mice, TNF signals via TNFR1 to induce TRADD-FADD-caspase-8-dependent apoptosis. (B) RIPK1 synergizes with RelA-dependent NF- $\kappa B$ signaling to prevent hepatocyte apoptosis under steadystate conditions. Neither RIPK1 $1^{\mathrm{LPC}-\mathrm{KO}}$ nor RelA ${ }^{\mathrm{LPC}-\mathrm{KO}}$ mice develop spontaneous liver disease. Mice lacking both RIPK1 and RelA in LPCs develop spontaneous hepatocyte apoptosis, chronic liver damage, and liver tumors. The spontaneous liver disease develops independently of TNF and is likely induced by yet unknown receptor(s) that activate apoptosis independently of RIPK1. (C) RIPK1 prevents early TNF-mediated hepatocyte apoptosis in response to DEN-induced DNA damage. Increased apoptosis of hepatocytes early on after DEN injection in RIPK1 ${ }^{\text {LPC-Ko }}$ mice likely eliminates cells carrying damaged DNA, resulting in reduced tumor development. TNFR1 deficiency prevents early hepatocyte apoptosis and restores normal DEN-induced tumorigenesis in RIPK1 ${ }^{\mathrm{LPC}-\mathrm{KO}}$ mice. death of RIPK1-deficient hepatocytes is consistent with the model of 2 different pathways inducing apoptosis downstream of TNFR1, one that is RIPK1 dependent and one that is TRADD dependent (39). Notably, FADD deficiency fully prevented TNF-induced death of RIPK1-deficient hepatocytes, showing that hepatocytes lacking RIPK1 die exclusively by apoptosis and are not susceptible to necroptosis. These results were supported by the finding that treatment with Z-VAD-FMK prevented TNF-induced apoptosis of either WT or RIPK1-deficient hepatocytes and did not sensitize these cells to necroptosis. This is in contrast with RIPK1-deficient embryos that die perinatally and are only rescued by combined inhibition of FADD-caspase-8-dependent apoptosis and RIPK3MLKL-dependent necroptosis (40-42), but also RIPK1-deficient keratinocytes that undergo RIPK3-MLKL-dependent necroptosis, triggering skin inflammation $(8,43)$. Therefore, apoptosis is the main pathway driving TNF-induced death in hepatocytes, likely due to the very low levels of RIPK3 expression in hepatocytes (44).

In our experiments, RIPK1 deficiency partly inhibited TNFinduced activation of NF- $\mathrm{kB}$, suggesting that reduced NF- $\mathrm{\kappa B}$ activation could contribute to the increased susceptibility of Ripk1knockout hepatocytes to TNF-mediated apoptosis. These results appear to be in contrast with the finding that knockdown or knockout of Ripk1 did not compromise TNF-induced NF- $\mathrm{kB}$ activation in hepatocytes $(20,22,24)$. This discrepancy is most likely due to differences in the quantitative assessment of NF- $\mathrm{\kappa B}$ signaling, as extensively discussed in previous studies on the role of RIPK1 in 
TNF-induced NF- $\kappa B$ activation in MEFs, where both essential and redundant functions of RIPK1 were proposed $(9,11)$. Collectively, these studies suggest that RIPK1 contributes to but is not essential for TNF-induced NF- $\kappa \mathrm{B}$ activation in hepatocytes.

While RIPK1 is critical for preventing endotoxin-induced hepatocyte death, it is not required for normal liver homeostasis, as RIPK1 $1^{\mathrm{LPC}-\mathrm{KO}}$ mice did not develop spontaneous liver pathology. $\mathrm{NF}-\kappa \mathrm{B}$ inhibition in LPCs also did not cause considerable spontaneous liver pathology, but sensitized the liver to endotoxininduced liver injury (16). However, mice lacking both RIPK1 and RelA in LPCs spontaneously developed hepatocyte apoptosis, chronic liver damage, and hepatocellular carcinoma, demonstrating that RIPK1 cooperates with RelA-dependent NF- $\mathrm{B}$ signaling to prevent hepatocyte apoptosis under physiological conditions. At first glance, these results contradict our findings that RIPK1 deficiency considerably inhibited TNF-induced NF- $\kappa \mathrm{B}$ activation in hepatocytes. However, TNFR1 deficiency did not prevent hepatocyte apoptosis, chronic hepatitis, and liver tumor development in RIPK1 ${ }^{\mathrm{LPC}-\mathrm{KO}}$ RelA ${ }^{\mathrm{LPC}-\mathrm{KO}}$ mice, showing that TNF-independent, as yet unidentified, mechanisms drive the spontaneous liver pathology in this model. The most likely explanation of these results is that NF- $\kappa \mathrm{B}$ activation by a stimulus that does not engage RIPK1 cooperates with NF- $\mathrm{BB}$-independent RIPK1 signaling to prevent spontaneous hepatocyte apoptosis and the development of chronic liver disease and cancer (Figure 8B). Interestingly, a recent study showed that mice with combined ablation of RIPK1 and TRAF2 in LPCs develop spontaneous chronic cholestatic liver disease and liver tumors, which are prevented by LPC-specific caspase- 8 knockout (20). The authors of this study suggested that RIPK1 and TRAF2 share redundant functions in regulating TNFR1-mediated hepatocyte apoptosis and liver cancer by NF- $\mathrm{BB}$-dependent and -independent functions. However, it remains unclear whether the liver disease in mice lacking RIPK1 and TRAF2 in LPCs is caused by TNF as suggested or whether this phenotype is independent of TNFR1 signaling, as in our RIPK1 $1^{\mathrm{LPC}-\mathrm{KO}} \mathrm{RelA}^{\mathrm{LPC}-\mathrm{KO}}$ mice.

The phenotype of RIPK $1^{\mathrm{LPC}-\mathrm{KO}} \mathrm{Rel} \mathrm{A}^{\mathrm{LPC}-\mathrm{KO}}$ mice is reminiscent of the liver pathology of NEMO ${ }^{\mathrm{LPC}-\mathrm{KO}}$ mice, which show TNFR1-independent hepatocyte apoptosis, chronic steatohepatitis, and HCC development $(16,17)$. Interestingly, inhibition of RIPK1 kinase activity prevented the liver pathology of $\mathrm{NEMO}^{\mathrm{LPC}-\mathrm{KO}}$ mice, but in the absence of RIPK1 protein, NEMO-deficient hepatocytes died by a TRADD-dependent mechanism that was sufficient to cause chronic liver disease and HCC $(16,17)$. Together, these findings highlight the complex kinase-dependent and -independent functions of RIPK1 in regulating hepatocyte survival and apoptosis. Kinase-independent RIPK1 functions cooperate with NF- $\kappa \mathrm{B}$ to prevent hepatocyte apoptosis. However, when NEMO is absent, RIPK1 kinase activity drives hepatocyte apoptosis, chronic liver disease, and cancer. Therefore, mechanisms regulating RIPK1 kinase-dependent and-independent functions play a critical role in liver homeostasis and disease. Previous studies suggested that IкB kinases directly phosphorylate RIPK1 to regulate its prosurvival functions $(23,45)$; however, it remains unclear whether NEMO regulates RIPK1 by facilitating its phosphorylation by IKKs.

RIPK1 deficiency reduced DEN-induced liver carcinogenesis in mice. The protective effect of RIPK1 deficiency was mediated by TNFR1, as mice with combined loss of RIPK1 and TNFR1 in LPCs were not protected from DEN-induced liver tumor development. RIPK1 $1^{\text {LPC-KO }}$ mice already showed increased caspase-8-mediated hepatocyte apoptosis 3 hours after DEN administration, which was diminished in RIPK1 $1^{\mathrm{LPC}-\mathrm{KO}}$ TNFR1 $1^{\mathrm{LPC}-\mathrm{KO}}$ mice, suggesting that TNFR1-dependent apoptosis of RIPK1-deficient hepatocytes could be at least in part responsible for reduced tumor development by eliminating cells carrying damaged DNA (Figure $8 \mathrm{C}$ ). In a previous study, increased DEN-induced apoptosis of IKK2-deficient hepatocytes was proposed to cause increased carcinogenesis by stimulating enhanced compensatory proliferation in the liver of mice with hepatocyte-specific IKK2 deficiency (34). However, the livers of DEN-injected RIPK $1^{\text {LPC-KO }}$ mice did not contain more proliferating cells compared with the livers of control mice; therefore, in these mice, the increased apoptosis did not stimulate increased compensatory proliferation of hepatocytes. Considering that the number of apoptotic hepatocytes in the livers of DEN-injected RIPK1 $1^{\mathrm{LPC}-\mathrm{KO}}$ mice is relatively small, it would be unlikely to result in a substantial reduction of liver mass that could trigger compensatory proliferation as observed in response to partial hepatectomy or other models of severe acute liver injury. At this stage, it remains unclear why deficiency of RIPK1 or IKK2 has opposing effects in the model of DEN-induced hepatocarcinogenesis, but these results suggest that RIPK1 regulates liver tumor development in this model by IKK/NF- $\mathrm{BB}$-independent mechanisms.

Our experiments in mice expressing kinase-inactive RIPK1 showed that RIPK1 kinase activity is not required for DEN-induced hepatocyte apoptosis and liver tumorigenesis, providing genetic evidence that kinase-independent RIPK1 functions promote DENinduced hepatocarcinogenesis. In addition, these results showed that ripoptosome-mediated cell death is not implicated in genotoxic stress-induced hepatocyte apoptosis and liver cancer development induced by DEN. These findings are interesting in view of previous studies suggesting that RIPK1-dependent apoptosis may be critical for the response of cells to genotoxic stress $(26,27)$.

Our in vivo experiments highlight the complex functions of RIPK1 in liver homeostasis and disease. RIPK1 scaffolding functions cooperate with $\mathrm{NF}-\kappa \mathrm{B}$ to prevent hepatocyte apoptosis, chronic liver disease, and cancer under steady-state conditions. However, in the absence of NEMO, RIPK1 is activated and drives hepatocyte apoptosis in a kinase-dependent manner, resulting in chronic liver disease and HCC development. In contrast, in the DEN model of hepatocarcinogenesis, where a single administration of DEN drives tumor development in the absence of chronic liver inflammation and disease, RIPK1 scaffolding functions support tumorigenesis by directing TNFR1 signaling toward a prosurvival tumor-promoting program. In summary, our results identify RIPK1 as a critical regulator of hepatocyte survival that cooperates with NF- $\kappa$ B to control TNFR1-dependent and -independent chronic liver inflammation and cancer.

\section{Methods}

Animals. Alfp-Cre (28), Ripk1 $1^{f / f l}$ (8), Ripk1 $1^{f / D 138 N}$ (33), Tnfri $1^{f / f l}$ (29), Fadd (31), $T r a d d^{f / f l}$ (30), and $R e l A^{f / f l}$ (46) mice were reported earlier. Mice were maintained at the specific pathogen-free animal facilities of the Institute for Genetics and the CECAD Research Center, University of Cologne, under a 12-hour dark/12-hour light cycle and were given a regular chow diet (Harlan diet no. 2918 or Prolab Isopro RMH3000 
5P76) ad libitum. For obesity-induced DEN carcinogenesis, animals were allowed ad libitum access to either NCD (sniff EF acc. D12450B [1] mod.) or HFD (ssniff EF acc. D12492 [1] mod.) starting at 4 weeks of age. Animals requiring medical attention were provided with appropriate care and excluded from the experiments described. No other exclusion criteria existed.

$D E N$-induced liver tumorigenesis. To induce liver tumorigenesis, mice were injected i.p. at 2 weeks of age with $25 \mathrm{mg} / \mathrm{kg}$ BW DEN (SigmaAldrich) and were sacrificed at the age of 32 or 36 weeks using $\mathrm{CO}_{2}$. Acute effects of DEN were studied using 6-week-old mice that were injected i.p. with $100 \mathrm{mg} / \mathrm{kg}$ BW DEN and were sacrificed at 3, 6, 12, 24, and 48 hours after DEN injection. For all experiments, only male mice were used.

Glucose tolerance test. Glucose tolerance tests were performed on animals fasted for 16 hours overnight. Mice were injected i.p. with 10 $\mu \mathrm{l} / \mathrm{g}$ BW glucose, and blood glucose levels were measured 15, 30, 60, and 120 minutes after glucose injection.

Measurement of serum parameters of liver damage. ALT, AST, LDL cholesterol, BILT, and alkaline phosphatase levels in the serum were measured using standard assays in a Roche-Cobas C111 Biochemical Analyzer.

Immunohistological analysis of liver sections. Paraffin sections were rehydrated, and heat-induced antigen retrieval was performed in TRIS-citrate buffer. Sections were incubated with primary antibodies for anti-CC3 (catalog 9661, Cell Signaling), anti-CC8 (catalog 8592, Cell Signaling), anti-Ki-67 (catalog M724901, Dako), anti- $\alpha$-SMA (catalog A2547, Sigma-Aldrich), anti-F4/80 (clone A3-1, AbD Serotec), anti- $\gamma \mathrm{H} 2 \mathrm{AX}$ (catalog 05-636, Millipore), anti-p53 (catalog NCL-p53CM5p, Leica), and anti-CK-19 (Developmental Studies Hybridoma Bank, TROMA-III). Biotinylated secondary antibodies were purchased from PerkinElmer and Dako. Stainings were visualized with ABC Kit Vectastain Elite (Vector Laboratories) and DAB substrate (DAKO and Vector Laboratories). Incubation times with DAB substrate were equal for all samples. For assessment of fibrosis, sections were stained with Picro Sirius Red Stain Kit (catalog ab150681, Abcam).

IHC quantification was performed on 5 to 10 randomly selected fields ( $\times 10$ magnification) per liver section. Liver sections from 3 to 7 mice per genotype were analyzed. $\mathrm{CC}^{+}, \mathrm{Ki}-67^{+}$, and $\mathrm{CC} 8^{+}$cells were counted manually. $\gamma \mathrm{H} 2 \mathrm{AX}^{+}$cells were quantified using Image (NIH) by applying the appropriate pixel threshold equally on all selected images. Graphs indicate the average number of $\gamma \mathrm{H} 2 \mathrm{AX}^{+}$cells of 5 randomly selected fields per mouse. Sirius red quantification was performed on 3 randomly selected high-power fields (HPF) per liver section. Three mice per genotype were analyzed. Quantification was performed using Image J and is expressed as percentage of stained area over total tissue area.

Macroscopic and histopathological evaluation of liver tumors. Livers of sacrificed mice were excised, digitally photographed with their dorsal side exposed, and weighed to calculate the liver/BW ratio. The tumor number and size were determined by counting the number of visible tumors/nodules and measuring their diameter. H\&E-stained liver sections were assessed for the presence and stage of HCC development in the following ascending order of severity: no pathology, clear cell foci, small and large cell changes (anisokaryosis), dysplastic foci, dysplastic nodules, and early, small, or well-differentiated HCC. Each liver sample was placed only based on the most advanced stages, despite the fact that less advanced stages were also observed in these liver samples. In the corresponding graphs, each bar represents the percentage of livers per genotype in which the indicated stage was identified as the most advanced disease stage.

Gene expression analysis with qRT-PCR. Total RNA was extracted with TRIzol Reagent (Life Technologies) and RNeasy Columns (QIAGEN) followed by cDNA preparation with Superscript III cDNA-Synthesis Kit (Life Technologies). qRT-PCR was performed with TaqMan probes in duplicate for each sample (Life Technologies) with TATA box-binding protein as reference gene. Relative expression of gene transcripts was analyzed by using the $2^{-\Delta \Delta C t}$ method.

Multiplex assay. The expression of TNF was measured using a bead-based multiplex immunoassay. $50 \mu \mathrm{l}$ of serum from Ripk1 $1^{f / f l}$ and RIPK1 $^{\text {LPC-KO }}$ mice was analyzed using the Bio-Rad Kit (Bio-Rad) in a multiplex analyzer (Bio-plex200, Bio-Rad) according to the manufacturer's instructions. The concentration was calculated from the standard curve and presented as $\mathrm{pg} / \mathrm{ml}$ of serum.

Immunoblotting. Liver tissues were homogenized using Precellys in protein lysis buffer supplemented with protease and phosphatase inhibitors (1 tablet per $10 \mathrm{ml}$ lysis buffer, Roche). Protein lysates $(50 \mu \mathrm{g} / 20 \mu \mathrm{l})$ were separated on SDS-PAGE and transferred to PVDF membranes (IPVH00010, Millipore). Membranes were blocked with $5 \%$ milk/0.1\% PBST and were probed with primary antibodies against $\beta$-actin (catalog sc-1616, Santa Cruz Biotechnology Inc.), CC3 (catalog 9661, Cell Signaling), cleaved PARP1 (catalog 9542, Cell Signaling),

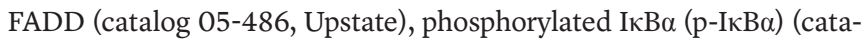
$\log 9246$, Cell Signaling), IкB $\alpha$ (catalog sc-371, Santa Cruz Biotechnology Inc.), lamin A/C (catalog sc-6215, Santa Cruz Biotechnology Inc.), RIPK1 (catalog 610459, BD Biosciences), RIPK3 (catalog ADI-905242-100, Enzo), anti-RelA (catalog sc-372, Santa Cruz Biotechnology Inc.) and p53 (catalog 2524, Cell Signaling), TNFR1 (catalog 13377, Cell Signaling), and TRADD (catalog sc-7868, Santa Cruz Biotechnology Inc.) for 16 hours at $4^{\circ} \mathrm{C}$. Membranes were washed with $0.1 \%$ PBST and were incubated with a horseradish peroxidase-conjugated anti-rabbit, anti-mouse (GE Healthcare and Jackson ImmuneResearch), anti-rat (catalog 112-035-003, Jackson Immuno Research), or anti-goat (catalog 705-035-003, Jackson Immuno Research) secondary antibody. Proteins were detected with enhanced chemiluminescent detection substrate (GE Healthcare and Thermo Scientific).

Subcellular fractionation. Primary hepatocytes were directly lysed in hypotonic buffer (50 mM Tris-HCl, pH 8.0, 2 mM EDTA, 10\% glycerol, and $0.1 \%$ Nonidet P-40) for 10 minutes on ice, followed by at 2,300 $g$ centrifugation at $4^{\circ} \mathrm{C}$ for 5 minutes. The supernatants were collected as cytoplasmic extracts. The pellets were washed twice with hypotonic buffer and lysed in $20 \mathrm{mM}$ HEPES-KOH, pH 7.6, $150 \mathrm{mM} \mathrm{NaCl}, 1.5 \mathrm{mM}$ $\mathrm{MgCl}_{2}, 1$ mM EGTA, 1\% Triton X-100, 10\% glycerol, PhosSTOP phosphatase inhibitors (Roche), and complete protease inhibitors (Roche) for 30 minutes on ice. Cell lysates were centrifuged for 10 minutes at $16,000 \mathrm{~g}$ at $4^{\circ} \mathrm{C}$, and the supernatants were collected as nuclear extracts.

Hepatocyte isolation. Primary hepatocytes were isolated from livers of 5-week-old mice by perfusing through the vena cava with Earle's balanced salt solution (EBSS) supplemented with 100 mM EGTA, followed by a collagenase solution containing EBSS, $15 \mathrm{mg}$ collagenase type 2, and $2 \mathrm{mg}$ trypsin inhibitor at $37^{\circ} \mathrm{C}$. Afterwards, the liver was gently scraped in DMEM medium supplemented with $1 \%$ FCS and filtered through a $70-\mu \mathrm{m}$ nylon filter. The flow-through was mixed with 1 volume of a $90 \%$ Percoll/HBSS solution and spun at $450 \mathrm{~g}$ for 7 minutes. Hepatocytes settled at the bottom were washed twice with DMEM 1\% FCS medium and were plated on collagen-coated plates. 
Primary hepatocytes were seeded on collagen-coated 6-well plates with $3 \times 10^{5}$ hepatocytes/well for immunoblot analysis and on 12-well plates with $1.5 \times 10^{5}$ hepatocytes/well for lactate dehydrogenase (LDH) assay. Four hours after plating, medium was changed.

LDH assay. Primary hepatocyte death in vitro was determined using an LDH release-based cytotoxicity assay (Promega) after incubating primary hepatocytes for 20 hours in the absence or presence of $20 \mu \mathrm{M}$ Z-VAD-FMK (Enzo LifeSciences), $10 \mu \mathrm{g} / \mathrm{ml}$ anti-TNF (Etanercept), or $50 \mathrm{ng} / \mathrm{ml}$ anti-FasL (106808, Biozol). LDH ratio (released/total LDH) was determined on cell sample supernatants before and after cell lysis using CytoTox 96 Cytotoxicity Assay (Promega) according to the manufacturer's protocol. The experiments were performed in triplicate.

Statistics. To determine the group size necessary for adequate statistical power, power analysis was performed using preliminary data sets. Statistical analysis of comparisons between 2 groups of nonparametric data was assessed by Mann-Whitney $U$ test. For multiple group comparison, 1-way ANOVA was used with a post-hoc Tukey's test for pairwise comparison of subgroups (equal variance assumed). The $\chi^{2}$ test was used for the comparison of tumor size distribution between 2 groups. Two-way ANOVA was used when 2 independent variables were compared. To compare the survival curves of mice, the Mantel-Cox test was used. A $P$ value of less than 0.05 was considered significant. Statistical analysis was performed with Prism version 6.0 (GraphPad).

Study approval. All animal procedures were conducted in accordance with European, national, and institutional guidelines, and protocols were approved by local government authorities (Landesamt für Natur, Umwelt, und Verbraucherschutz, Nordrhein-Westfalen, Germany).

\section{Author contributions}

TMV, AP, and VK performed genetic crosses, tissue sampling, data collection, and interpretation. NP and TMV performed serum TNF measurements. BKS performed histopathological analysis of mouse livers. MP conceived and coordinated the project and wrote the manuscript together with TMV, AP, and VK.

\section{Acknowledgments}

We thank G. Kollias for providing Tnfr $1^{f l f l}$ mice and J. Kuth, C. Uthoff-Hachenberg, E. Mahlberg, B. Kühnel, and E. Stade for technical assistance. Research reported in this publication was supported by grants from Worldwide Cancer Research (award no. 15-0228) and from the European Research Council (grant agreement no. 323040 to MP). TMV was supported by a fellowship from the Cologne Graduate School of Ageing Research, and VK was supported by a Marie Curie Career Development Fellowship (FP7-PEOPLE-2010-IEF; proposal no. 275767).

Address correspondence to: Manolis Pasparakis, CECAD Research Center, Institute for Genetics, University of Cologne, JosephStelzmann-Straße 26, D-50931 Cologne, Germany. Phone: 49.221.478.84349; Email: pasparakis@uni-koeln.de.
1. Shariff MI, Cox IJ, Gomaa AI, Khan SA, Gedroyc W, Taylor-Robinson SD. Hepatocellular carcinoma: current trends in worldwide epidemiology, risk factors, diagnosis and therapeutics. Expert Rev Gastroenterol Hepatol. 2009;3(4):353-367.

2. El-Serag HB. Hepatocellular carcinoma. $N$ Engl J Med. 2011;365(12):1118-1127.

3. Wang K. Molecular mechanisms of hepatic apoptosis. Cell Death Dis. 2014;5:e996.

4. Schattenberg JM, Schuchmann M, Galle PR. Cell death and hepatocarcinogenesis: Dysregulation of apoptosis signaling pathways. J Gastroenterol Hepatol. 2011;26 Suppl 1:213-219.

5. Pasparakis M, Vandenabeele P. Necroptosis and its role in inflammation. Nature. 2015;517(7534):311-320.

6. Micheau O, Tschopp J. Induction of TNF receptor I-mediated apoptosis via two sequential signaling complexes. Cell. 2003;114(2):181-190.

7. Walczak H, Iwai K, Dikic I. Generation and physiological roles of linear ubiquitin chains. BMC Biol. 2012;10:23.

8. Dannappel M, et al. RIPK1 maintains epithelial homeostasis by inhibiting apoptosis and necroptosis. Nature. 2014;513(7516):90-94.

9. Kelliher MA, Grimm S, Ishida Y, Kuo F, Stanger BZ, Leder P. The death domain kinase RIP mediates the TNF-induced NF-kappaB signal. Immunity. 1998;8(3):297-303.

10. Takahashi N, et al. RIPK1 ensures intestinal homeostasis by protecting the epithelium against apoptosis. Nature. 2014;513(7516):95-99.

11. Wong WW, Gentle IE, Nachbur U, Anderton $\mathrm{H}$, Vaux DL, Silke J. RIPK1 is not essential for TNFR1-induced activation of NF-kappaB. Cell Death Differ. 2010;17(3):482-487.
12. Ben-Neriah Y, Karin M. Inflammation meets cancer, with NF- $\kappa \mathrm{B}$ as the matchmaker. Nat Immu nol. 2011;12(8):715-723.

13. Hayden MS, Ghosh S. NF- $\kappa$ B, the first quartercentury: remarkable progress and outstanding questions. Genes Dev. 2012;26(3):203-234.

14. Luedde T, Schwabe RF. NF- $\kappa$ B in the liver--linking injury, fibrosis and hepatocellular carcinoma. Nat Rev Gastroenterol Hepatol. 2011;8(2):108-118.

15. Sun B, Karin M. NF-kappaB signaling, liver disease and hepatoprotective agents. Oncogene. 2008;27(48):6228-6244.

16. Kondylis V, et al. NEMO prevents steatohepatitis and hepatocellular carcinoma by inhibiting RIPK1 kinase activity-mediated hepatocyte apoptosis. Cancer Cell. 2015;28(5):582-598.

17. Luedde T, et al. Deletion of NEMO/IKKgamma in liver parenchymal cells causes steatohepatitis and hepatocellular carcinoma. Cancer Cell. 2007;11(2):119-132.

18. Liedtke C, et al. Loss of caspase-8 protects mice against inflammation-related hepatocarcinogenesis but induces non-apoptotic liver injury. Gastroenterology. 2011;141(6):2176-2187.

19. Ehlken H, et al. Death receptor-independent FADD signalling triggers hepatitis and hepatocellular carcinoma in mice with liver parenchymal cell-specific NEMO knockout. Cell Death Differ. 2014;21(11):1721-1732.

20. Schneider AT, et al. RIPK1 suppresses a TRAF2dependent pathway to liver cancer. Cancer Cell. 2017;31(1):94-109.

21. Filliol A, et al. RIPK1 protects hepatocytes from Kupffer cells-mediated TNF-induced apoptosis in mouse models of PAMP-induced hepatitis. J Hepatol. 2017;66(6):1205-1213.
22. Suda J, et al. Knockdown of RIPK1 markedly exacerbates murine immune-mediated liver injury through massive apoptosis of hepatocytes, independent of necroptosis and inhibition of NF-кB. JImmunol. 2016;197(8):3120-3129.

23. Koppe $\mathrm{C}$, et al. I $\kappa \mathrm{B}$ kinase $\alpha / \beta$ control biliary homeostasis and hepatocarcinogenesis in mice by phosphorylating the cell-death mediator receptor-interacting protein kinase 1 . Hepatology. 2016;64(4):1217-1231.

24. Filliol A, et al. RIPK1 protects from TNF- $\alpha$ mediated liver damage during hepatitis. Cell Death Dis. 2016;7(11):e2462.

25. Yang Y, et al. A cytosolic ATM/NEMO/RIP1 complex recruits TAK1 to mediate the NF-kappaB and p38 mitogen-activated protein kinase (MAPK)/ MAPK-activated protein 2 responses to DNA damage. Mol Cell Biol. 2011;31(14):2774-2786.

26. Biton S, Ashkenazi A. NEMO and RIP1 control cell fate in response to extensive DNA damage via TNF- $\alpha$ feedforward signaling. Cell. 2011;145(1):92-103.

27. Tenev T, et al. The Ripoptosome, a signaling platform that assembles in response to genotoxic stress and loss of IAPs. Mol Cell. 2011;43(3):432-448.

28. Kellendonk C, Opherk C, Anlag K, Schütz G, Tronche F. Hepatocyte-specific expression of Cre recombinase. Genesis. 2000;26(2):151-153.

29. Van Hauwermeiren F, et al. Safe TNF-based antitumor therapy following p55TNFR reduction in intestinal epithelium. J Clin Invest. 2013;123(6):2590-2603.

30. Ermolaeva MA, et al. Function of TRADD in tumor necrosis factor receptor 1 signaling and in TRIF-dependent inflammatory responses. $\mathrm{Nat}$ 
Immunol. 2008;9(9):1037-1046.

31. Mc Guire C, et al. Oligodendrocyte-specific FADD deletion protects mice from autoimmunemediated demyelination. JImmunol. 2010;185(12):7646-7653.

32. Verna L, Whysner J, Williams GM. N-nitrosodiethylamine mechanistic data and risk assessment: bioactivation, DNA-adduct formation, mutagenicity, and tumor initiation. Pharmacol Ther. 1996;71(1-2):57-81.

33. Polykratis A, et al. Cutting edge: RIPK1 kinase inactive mice are viable and protected from TNF-induced necroptosis in vivo. J Immunol. 2014;193(4):1539-1543.

34. Maeda S, Kamata H, Luo JL, Leffert H, Karin M. IKKbeta couples hepatocyte death to cytokinedriven compensatory proliferation that promotes chemical hepatocarcinogenesis. Cell. 2005;121(7):977-990.

35. Lukas J, Lukas C, Bartek J. More than just a focus: The chromatin response to DNA damage and its role in genome integrity maintenance. Nat Cell Biol. 2011;13(10):1161-1169.

36. Bieging KT, Mello SS, Attardi LD. Unravelling mechanisms of p53-mediated tumour suppression. Nat Rev Cancer. 2014;14(5):359-370.

37. Nakagawa $\mathrm{H}$, et al. ER stress cooperates with hypernutrition to trigger TNF-dependent spontaneous HCC development. Cancer Cell. 2014;26(3):331-343.

38. Park EJ, et al. Dietary and genetic obesity promote liver inflammation and tumorigenesis by enhancing IL-6 and TNF expression. Cell. 2010;140(2):197-208.

39. Wang L, Du F, Wang X. TNF-alpha induces two distinct caspase-8 activation pathways. Cell. 2008;133(4):693-703.

40. Rickard JA, et al. RIPK1 regulates RIPK3-MLKLdriven systemic inflammation and emergency hematopoiesis. Cell. 2014;157(5):1175-1188.

41. Kaiser WJ, et al. RIP1 suppresses innate immune necrotic as well as apoptotic cell death during mammalian parturition. Proc Natl Acad Sci U S A. 2014;111(21):7753-7758.

42. Dillon CP, et al. RIPK1 blocks early postnatal lethality mediated by caspase- 8 and RIPK3. Cell. 2014;157(5):1189-1202.

43. Lin J, et al. RIPK1 counteracts ZBP1-mediated necroptosis to inhibit inflammation. Nature. 2016;540(7631):124-128.

44. Dara L, et al. Receptor interacting protein kinase 1 mediates murine acetaminophen toxicity independent of the necrosome and not through necroptosis. Hepatology. 2015;62(6):1847-1857.

45. Dondelinger $\mathrm{Y}$, et al. NF- $\kappa \mathrm{B}$-independent role of IKK $\alpha /$ IKK $\beta$ in preventing RIPK1 kinase-dependent apoptotic and necroptotic cell death during TNF signaling. Mol Cell. 2015;60(1):63-76.

46. Luedde T, Heinrichsdorff J, de Lorenzi R, De Vos R, Roskams T, Pasparakis M. IKK1 and IKK2 cooperate to maintain bile duct integrity in the liver. Proc Natl Acad Sci US A. 2008;105(28):9733-9738. 